\title{
Bases for certain cohomology representations of the symmetric group
}

\author{
Anthony Henderson
}

Received: 12 August 2005 / Accepted: 10 March 2006/

Published online: 11 July 2006

(C) Springer Science + Business Media, LLC 2006

\begin{abstract}
We give a combinatorial description (including explicit differential-form bases) for the cohomology groups of the space of $n$ distinct nonzero complex numbers, with coefficients in rank-one local systems which are of finite monodromy around the coordinate hyperplanes and trivial monodromy around all other hyperplanes. In the case where the local system is equivariant for the symmetric group, we write the cohomology groups as direct sums of inductions of one-dimensional characters of subgroups. This relies on an equivariant description of the Orlik-Solomon algebras of full monomial reflection groups (wreath products of the symmetric group with a cyclic group). The combinatorial models involved are certain representations of these wreath products which possess bases indexed by labelled trees.
\end{abstract}

Keywords Hyperplane complement · Cohomology · Representation · Symmetric group

\section{Introduction}

Fix a positive integer $n$. This paper concerns the cohomology of the complex hyperplane complement

$$
T(1, n):=\left\{\left(z_{1}, z_{2}, \ldots, z_{n}\right) \in \mathbb{C}^{n} \mid z_{i} \neq 0, \forall i, z_{i} \neq z_{j}, \forall i \neq j\right\}
$$

Let $\left(\Omega^{\bullet}(T(1, n)), d\right)$ be the differential graded algebra of regular differential forms on $T(1, n)$. The Orlik-Solomon algebra $A^{\bullet}(T(1, n))$ is defined to be the $\mathbb{C}$-subalgebra of

This work was supported by Australlian Research Council grant DP0344185.

A. Henderson

School of Mathematics and Statistics, University of Sydney, NSW 2006, Australia

e-mail: anthonyh@maths.usyd.edu.au 
$\Omega^{\bullet}(T(1, n))$ generated by the following closed 1 -forms corresponding to the hyperplanes:

$$
\omega_{i}:=\frac{d z_{i}}{z_{i}}, \forall i, \quad \text { and } \quad \omega_{i, j}:=\frac{d z_{i}-d z_{j}}{z_{i}-z_{j}}, \forall i<j
$$

A famous result of Brieskorn (valid for all hyperplane complements) states that the inclusion $\left(A^{\bullet}(T(1, n)), 0\right) \hookrightarrow\left(\Omega^{\bullet}(T(1, n)), d\right)$ is a quasi-isomorphism of cochain complexes, so $A^{p}(T(1, n)) \cong H^{p}(T(1, n), \mathbb{C})$ for all $p$. Moreover, results of Arnold (in this case) and Orlik and Solomon (for general hyperplane complements) give a combinatorial description of $A^{\bullet}(T(1, n))$, including the following explicit basis. We say that a rooted forest with vertex set $\{1, \ldots, n\}$ is rectified if the root of each tree is its largest vertex, and the path from each other vertex to the root is an increasing sequence. Let $\mathcal{F}(1, n)^{\circ}$ be the set of such rectified forests equipped with a partition of the set of roots into two subsets, called open and closed. To each $F \in \mathcal{F}(1, n)^{\circ}$ associate the form $\alpha(F) \in A^{\bullet}(T(1, n))$ which is the wedge product of all $\omega_{i, j}$ for edges $i-j$ of $F$ and all $\omega_{i}$ for closed roots $i$ of $F$. (For now, we leave the order of factors in the wedge product unspecified, so $\alpha(F)$ is defined only up to sign.) Then $\left\{\alpha(F) \mid F \in \mathcal{F}(1, n)^{\circ}\right\}$ is a basis of $A^{\bullet}(T(1, n)$ ) (see Theorem 3.2 below).

Now suppose we replace the complex coefficients in this cohomology by a general rank-one local system on $T(1, n)$. Any element $\omega=\sum_{i} a_{i} \omega_{i}+\sum_{i<j} a_{i, j} \omega_{i, j}$ of $A^{1}(T(1, n))$ determines such a local system $\mathcal{L}_{\omega}$, whose local sections are the solutions of the differential equation $d f+f \omega=0$. We have obvious isomorphisms $\mathcal{L}_{\omega} \otimes \mathcal{L}_{\omega^{\prime}} \cong \mathcal{L}_{\omega+\omega^{\prime}}$ for all $\omega, \omega^{\prime} \in A^{1}(T(1, n))$, and $\mathcal{L}_{\omega} \cong \mathcal{L}_{0}=\mathbb{C}$ for $\omega \in \mathbb{Z}\left\{\omega_{i}, \omega_{i, j}\right\}$. If $\ell_{\omega}: \Omega^{\bullet}(T(1, n)) \rightarrow \Omega^{\bullet}(T(1, n))$ denotes the map $\alpha \mapsto \omega \wedge \alpha$, then we have a natural isomorphism

$$
H^{p}\left(T(1, n), \mathcal{L}_{\omega}\right) \cong \frac{\operatorname{ker}\left(d+\ell_{\omega}: \Omega^{p}(T(1, n)) \rightarrow \Omega^{p+1}(T(1, n))\right)}{\operatorname{im}\left(d+\ell_{\omega}: \Omega^{p-1}(T(1, n)) \rightarrow \Omega^{p}(T(1, n))\right)} .
$$

For most values of $\omega$, this cohomology too can be described in terms of the OrlikSolomon algebra, by a general result of Esnault, Schechtman, and Viehweg, sharpened by Schechtman, Terao, and Varchenko. We say that $\omega=\sum_{i} a_{i} \omega_{i}+\sum_{i<j} a_{i, j} \omega_{i, j}$ is resonant if any of the following holds:

- $\sum_{i} a_{i}+\sum_{i<j} a_{i, j}$ is a nonzero integer; or

- $\sum_{i<j, i, j \in I} a_{i, j}$ is a positive integer, for some subset $I \subseteq\{1, \ldots, n\}$; or

- $\sum_{i \in I} a_{i}+\sum_{i<j, i, j \in I} a_{i, j}$ is a positive integer, for some proper subset $I \subset$ $\{1, \ldots, n\}$.

The following result is a special case of [14, Theorem 9] (see also the survey articles [12] and [15]). 
Theorem 1.1. If $\omega$ is not resonant, the inclusion $\left(A^{\bullet}(T(1, n)), \ell_{\omega}\right) \hookrightarrow$ $\left(\Omega^{\bullet}(T(1, n)), d+\ell_{\omega}\right)$ is a quasi-isomorphism of cochain complexes, so

$$
\frac{\operatorname{ker}\left(\ell_{\omega}: A^{p}(T(1, n)) \rightarrow A^{p+1}(T(1, n))\right)}{\operatorname{im}\left(\ell_{\omega}: A^{p-1}(T(1, n)) \rightarrow A^{p}(T(1, n))\right)} \cong H^{p}\left(T(1, n), \mathcal{L}_{\omega}\right) .
$$

(Note that Brieskorn's result is the special case $\omega=0$.) Even if $\omega$ itself is resonant, it is often possible to replace it with a non-resonant element of $\omega+\mathbb{Z}\left\{\omega_{i}, \omega_{i, j}\right\}$, giving an isomorphic local system. However, there exist $\omega$ such that all elements of $\omega+\mathbb{Z}\left\{\omega_{i}, \omega_{i, j}\right\}$ are resonant: for instance, it is easy to see that $\frac{s}{n} \sum_{i} \omega_{i}$ has this property when $s \in \mathbb{Z}$ and $1<\operatorname{gcd}(s, n)<n$. The corresponding local systems cannot be handled by Theorem 1.1.

One result of this paper is an explicit basis of $H^{\bullet}\left(T(1, n), \mathcal{L}_{\sum a_{i} \omega_{i}}\right)$ where all $a_{i} \in \mathbb{Q}$ (note that the coefficients of all $\omega_{i, j}$ 's here are zero). This basis is given by differential forms attached to the subset of $\mathcal{F}(1, n)^{\circ}$ consisting of forests which satisfy

$$
\sum_{i \in T} a_{i} \in \mathbb{Z}, \quad \text { for all trees } T \text {. }
$$

(Note that if $\sum_{i=1}^{n} a_{i} \notin \mathbb{Z}$, this subset is empty.) The associated differential form $\bar{\beta}(F) \in \Omega^{\bullet}(T(1, n))$ is usually not in $A^{\bullet}(T(1, n))$, because there are two changes from the above definition of $\alpha(F)$. Firstly, any edge $i-j$ which is unbreakable, in the sense that deleting it results in a forest which no longer satisfies (1.2), contributes to the wedge product not $\omega_{i, j}$ but rather $\frac{z_{j} d z_{i}-z_{i} d z_{j}}{z_{i} z_{j}\left(z_{i}-z_{j}\right)}$. Secondly, the wedge product must be multiplied by a certain monomial in the $z_{i}$ 's, to be defined in Section 4 . The result (see Theorem 4.5 and Corollary 4.6) is that each such $\bar{\beta}(F)$ lies in $\operatorname{ker}\left(d+\ell_{\sum a_{i} \omega_{i}}\right)$, and their images in $H^{\bullet}\left(T(1, n), \mathcal{L}_{\sum a_{i} \omega_{i}}\right.$ ) form a basis. A consequence (Corollary 4.10) is that the $H^{\bullet}(T(1, n), \mathbb{C})$-module $H^{\bullet}\left(T(1, n), \mathcal{L}_{\sum a_{i} \omega_{i}}\right)$ is generated by those $\bar{\beta}(F)^{\text {'s }}$ which correspond to forests in which all roots are open and all edges are unbreakable. See Example 4.8 for the special case where all $a_{i}=\frac{s}{n}$ and $\operatorname{gcd}(s, n)=1$, which can also be handled by a result of Kawahara.

The special property of the local systems $\mathcal{L}_{\sum a_{i} \omega_{i}}, a_{i} \in \mathbb{Q}$, which makes such results possible (indeed, easy) is that they become trivial on pulling back to a covering space of $T(1, n)$ which is also a hyperplane complement. Namely, let $r$ be a positive integer such that $r a_{i} \in \mathbb{Z}$ for all $i$, and define

$$
T(r, n):=\left\{\left(z_{1}, z_{2}, \ldots, z_{n}\right) \in \mathbb{C}^{n} \mid z_{i} \neq 0, \forall i, z_{i}^{r} \neq z_{j}^{r}, \forall i \neq j\right\}
$$

The map $\varphi: T(r, n) \rightarrow T(1, n):\left(z_{1}, \ldots, z_{n}\right) \mapsto\left(z_{1}^{r}, \ldots, z_{n}^{r}\right)$ is an unramified Galois covering with group $\mu_{r}^{n}$, where $\mu_{r}$ is the cyclic group consisting of all complex $r$ th roots of 1 . The local system $\mathcal{L}_{\sum a_{i} \omega_{i}}$ is the direct summand of the pushforward $\varphi_{*}(\mathbb{C})$ corresponding to the character $\left(\zeta_{1}, \ldots, \zeta_{n}\right) \mapsto \zeta_{1}^{-r a_{1}} \ldots \zeta_{n}^{-r a_{n}}$ of $\mu_{r}^{n}$, so $H^{p}\left(T(1, n), \mathcal{L}_{\sum a_{i} \omega_{i}}\right)$ is isomorphic via the pull-back $\varphi^{*}$ to the corresponding isotypic component of $H^{p}(T(r, n), \mathbb{C})$ (for more details on this isomorphism, see Proposition 
4.1). The Orlik-Solomon results on $A^{\bullet}(T(r, n))$ lead easily to a basis $\{\beta(F)\}$ of this isotypic component, and a short calculation shows that this is the pull-back of $\{\bar{\beta}(F)\}$.

In fact I knew the basis $\{\beta(F)\}$ on $T(r, n)$ first, and was surprised to find a relatively nice formula for the corresponding basis on $T(1, n)$. However, one of the referees has suggested an alternative approach which avoids (explicitly) passing to the covering space, and thus removes some of the surprise. Namely, since the local system $\mathcal{L}_{\sum a_{i} \omega_{i}}$ has trivial monodromy around the hyperplanes $z_{i}=z_{j}$, one could successively delete these using the deletion-restriction result of Cohen ([2, Theorem 4]), and relate $H^{\bullet}\left(T(1, n), \mathcal{L}_{\sum a_{i} \omega_{i}}\right)$ to $H^{\bullet}\left(T(1, m), \mathcal{L}_{\sum a_{i} \omega_{f(i)}}\right)$ for various $m<n$ and maps $f:\{1, \ldots, n\} \rightarrow\{1, \ldots, m\}$; the rectified forests and the integrality condition (1.2) would reappear. Essentially, what this means is that one can mimic on the base space the steps of the proof of the Orlik-Solomon results on the covering space.

If this basis result had been the only goal in view, this paper would have been much shorter. But after the paper [11] of Lehrer and Solomon, one has a right to expect of such Orlik-Solomon-style descriptions that they take into account the action of the relevant symmetry group. In the present case, the symmetric group $S_{n}$ acts on $T(1, n)$ by permuting coordinates, and each cohomology group $H^{p}\left(T(1, n), \mathcal{L}_{\sum a_{i} \omega_{i}}\right)$ is thus a representation for the subgroup of $S_{n}$ which fixes $\sum a_{i} \omega_{i}$, namely

$$
Z_{\left(a_{i}\right)}:=\left\{w \in S_{n} \mid a_{w(i)}=a_{i}, \forall i\right\}
$$

On the model of $[11,(4.5)]$, one should aim to write this representation as a direct sum of inductions of one-dimensional characters of subgroups. Corollary 4.16 below accomplishes this in the case where all $a_{i}$ are equal, so $Z_{\left(a_{i}\right)}=S_{n}$; having understood this case, the interested reader will have no trouble imagining the general result. The reason for concentrating on this case (apart from simplifying the notation) is that it arose in [6], and the motivation for all this work was to give a better explanation for the following isomorphism of representations of $S_{n}$, which I originally proved by computing characters:

$$
H^{p}\left(T(1, n), \mathcal{L}_{\frac{s}{r} \sum \omega_{i}}\right) \cong \varepsilon_{n} \otimes \operatorname{Ind}_{W(r, n / r)}^{S_{n}}\left(\operatorname{det}_{n / r} \otimes H^{p-n+n / r}(T(r, n / r), \mathbb{C})\right)
$$

Here $\operatorname{gcd}(s, r)=1$ (so the fraction $\frac{s}{r}$ is in lowest terms), $\varepsilon_{n}$ is the sign character of $S_{n}, W(r, m)$ is the wreath product $\mu_{r} 2 S_{m}$, and $\operatorname{det}_{m}$ is the determinant of the natural representation of $W(r, m)$ on $\mathbb{C}^{m}$. For the new explanation, see Corollary 4.17.

In order to study these representations of $S_{n}$, we need a sufficiently equivariant description of $A^{\bullet}(T(r, n))$, one which takes into account the action of $W(r, n)$. Section 3 is devoted to such a description; in Corollary 3.12 we write $A^{\bullet}(T(r, n))$ as a direct sum of inductions of one-dimensional characters of subgroups of $W(r, n)$, generalizing a result of Douglass $([3,(1.1)])$ in the $r=2$ case. Since the Orlik-Solomon basis is not stable under $W(r, n)$, our approach is to consider the collection of all $W(r, n)$ translates of basis elements and the linear dependence relations they satisfy. Being suitably careful with the signs, we will find that these relations can be described in a uniform combinatorial way, using rooted forests which are now not necessarily rectified. This motivates the preparatory Section 2, where we study a representation 
of $W(r, n)$ defined abstractly by certain relations between labelled trees, inspired by [11] and [1].

\section{Representations of wreath products on trees}

Fix positive integers $r$ and $n$. As in the introduction, let $\mu_{r}$ denote the group of $r$ th complex roots of $1, S_{n}$ the group of permutations of $\{1, \ldots, n\}$, and $W(r, n)$ the wreath product $\mu_{r} 2 S_{n}$, of cardinality $r^{n} n$ !. In other words, $W(r, n)$ is the semidirect product $\mu_{r}^{n} \rtimes S_{n}$, where $S_{n}$ acts on $\mu_{r}^{n}$ by permuting the factors; we will usually write its elements in the form $\left(\zeta_{1}, \ldots, \zeta_{n}\right) w$, where $\zeta_{i} \in \mu_{r}$ and $w \in S_{n}$. Often we will abbreviate $\left(\zeta_{1}, \ldots, \zeta_{n}\right)$ as $\underline{\zeta}$. We need some one-dimensional characters of $W(r, n)$ :

- $\varepsilon_{n}$ defined by $\varepsilon_{n}(\zeta w)=\varepsilon_{n}(w)$, the extension of the sign character of $S_{n}$;

- $\operatorname{prod}_{n}$ defined by $\operatorname{prod}_{n}(\underline{\zeta} w)=\zeta_{1} \cdots \zeta_{n}$; and

- $\operatorname{det}_{n}:=\varepsilon_{n} \operatorname{prod}_{n}$.

The last is so named because it is the determinant arising from the standard representation of $W(r, n)$ on $\mathbb{C}^{n}$, in which $w$ is represented by the usual permutation matrix and $\underline{\zeta}$ by the diagonal matrix with entries $\left(\zeta_{1}, \ldots, \zeta_{n}\right)$. The image of $W(r, n)$ under this representation is the monomial reflection group $G(r, 1, n)$.

In this section we study a representation of $W(r, n)$ which has a basis indexed by certain labelled trees. Here are our conventions.

Definition 2.1. A directed tree on a nonempty finite set $I$ is a directed graph $T$ with vertex set $I$, containing no loops or cycles, such that there is exactly one vertex, the root, with out-degree 0 , and every other vertex has out-degree 1 .

It is clear that these conditions force the underlying graph of $T$ to be a tree, and that all edges are directed 'towards the root'. So by a well known result, the total number of directed trees on $I$ is $|I|^{|I|-1}$.

Definition 2.2. We define a $\mu_{r}$-labelled directed tree on $I$ to be a directed tree on $I$ equipped with a labelling of each edge by an element of $\mu_{r}$. (If $r=1$, the labels are all 1 and may be neglected.) If $T$ is a $\mu_{r}$-labelled directed tree, we write $i \stackrel{\eta}{\rightarrow} j$ (with a subscript $T$ when necessary) to mean that $i, j \in I$ and there is an edge from $i$ to $j$ in $T$ whose label is $\eta \in \mu_{r}$. Let $\mathcal{T}(r, I)$ be the set of $\mu_{r}$-labelled directed trees on $I$, and write $\mathcal{T}(r, n)$ for $\mathcal{T}(r,\{1, \ldots, n\})$.

Clearly the cardinality of $\mathcal{T}(r, n)$ is $(r n)^{n-1}$. We have a natural action of $W(r, n)$ on $\mathcal{T}(r, n)$, in which $S_{n}$ acts by permuting the vertex set and $\zeta \in \mu_{r}^{n}$ acts by multiplying the label of an edge from $i$ to $j$ by $\zeta_{i} \zeta_{j}^{-1}$. More formally, the following equivalences hold:

$$
i \underset{T}{\stackrel{\eta}{\longrightarrow}} j \Longleftrightarrow i \underset{\underline{\zeta . T}}{\stackrel{\eta \zeta_{i} \zeta_{j}^{-1}}{\longrightarrow}} j \Longleftrightarrow w(i) \underset{w . T}{\stackrel{\eta}{\longrightarrow}} w(j)
$$


for all $\underline{\zeta} \in \mu_{r}^{n}$ and $w \in S_{n}$. Linearizing, we obtain a representation of $W(r, n)$ on the vector space $\mathbb{C} \mathcal{T}(r, n)$ with $\mathcal{T}(r, n)$ as basis.

Now we want to define a quotient $\mathcal{V}(r, n)$ of $\mathbb{C} \mathcal{T}(r, n)$ by imposing some linear relations which the images [T] of $T \in \mathcal{T}(r, n)$ must satisfy. (The motivation for these relations will become clear when we consider differential forms in the next section.) The relations are of the following two kinds:

$$
\left[T_{1}\right]+\left[T_{2}\right]=\left[T_{3}\right]
$$

if $T_{1}, T_{2}, T_{3} \in \mathcal{T}(r, n)$ are identical except for the edges between three vertices $i, j$, and $k$, where we have

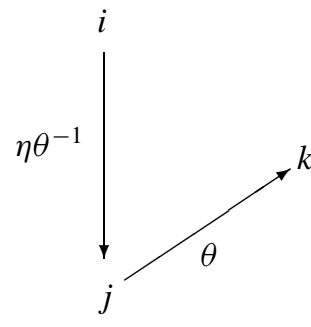

$T_{1}$

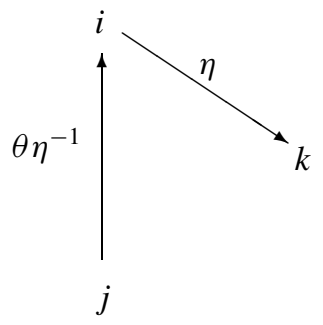

$T_{2}$

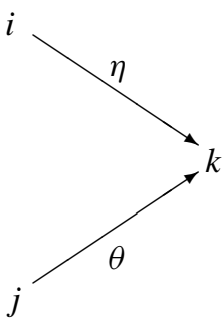

$T_{3}$

for some $\eta, \theta \in \mu_{r}$; and

$$
[T]+\left[T^{\prime}\right]=0,
$$

if $T, T^{\prime} \in \mathcal{T}(r, n)$ are identical except for the edge between two vertices $i$ and $j$, where we have:

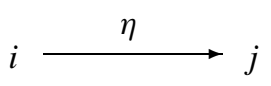

$T$

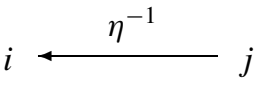

$T^{\prime}$

for some $\eta \in \mu_{r}$. (Note that this implies that $j$ is the root of $T$ and $i$ is the root of $T^{\prime}$.) It is clear that $W(r, n)$ permutes these relations, so we obtain a representation of $W(r, n)$ on $\mathcal{V}(r, n)$.

One feature of these relations is that they allow us to express any tree as a linear combination of those where the edges are always directed towards the greater vertex, a process of 'rectification' which is implicit in [11] and [1]. This is encapsulated in the next definition and result.

Definition 2.3. A tree $T \in \mathcal{T}(r, n)$ is rectified if $i \stackrel{\eta}{\rightarrow} j$ implies $i<j$. Let $\mathcal{T}(r, n)^{\circ}$ be the set of rectified trees. 
Note that $\mathcal{T}(r, n)^{\circ}$ has exactly $r^{n-1}(n-1)$ ! elements: the root must be $n$, and for $i<n$, there are $n-i$ possibilities for the end of the edge which starts at $i$, with $r$ possible labels for the edge.

Lemma 2.4. $\mathcal{V}(r, n)$ is spanned by $\left\{[T] \mid T \in \mathcal{T}(r, n)^{\circ}\right\}$.

Proof: By using relations of type (2.3) repeatedly to shift the root, we can express any [ $T$ ] for $T \in \mathcal{T}(r, n)$ as $\pm\left[T^{\prime}\right]$ where $T^{\prime}$ has root $n$. Now suppose that $T \in \mathcal{T}(r, n)$ has root $n$ and is unrectified. Let $a(T) \leq n-1$ be the maximal $i$ such that $T$ has an edge $i \stackrel{\eta}{\rightarrow} j$ where $i>j$, and let $b(T)$ be the length of the path from $a(T)$ to $n$ in $T$. It suffices to show that $[T]$ can be written as a linear combination of those $\left[T^{\prime}\right]$ where $T^{\prime}$ has root $n$ and is either rectified or has a pair $\left(a\left(T^{\prime}\right), b\left(T^{\prime}\right)\right)$ which precedes $(a(T), b(T))$ in lexicographic order. For this, define $T_{2}, T_{3} \in \mathcal{T}(r, n)$ so that $T, T_{2}, T_{3}$ form a triple as in (2.2), where $i=a(T), j$ is the end of the edge starting at $i$ in $T$, and $k$ is the end of the edge starting at $j$ in $T$ ( $j$ cannot be the root $n$, since by assumption it is less than $i$ ). Since $T_{2}$ and $T_{3}$ clearly have the same root as $T$, it suffices to show that they are either rectified or have an $(a, b)$ pair preceding that of $T$. Now if $k>a(T)=i$, then the edge starting with $i$ in both $T_{2}$ and $T_{3}$ is directed towards the greater vertex, so they are either rectified or have an $a$-value strictly less than $a(T)$. On the other hand, if $k<a(T)$, then $a\left(T_{2}\right)=a\left(T_{3}\right)=a(T)$, but $b\left(T_{2}\right)=b\left(T_{3}\right)=b(T)-1$, since $k$ is a step closer to the root than $j$ is in $T$. So in either case we are done.

Now the representation $\mathcal{V}(1, n)$ of $S_{n}$ has a convenient 'concrete' realization. For $T \in \mathcal{T}(1, n)$, define the polynomial $p_{T} \in \mathbb{C}\left[z_{1}, \ldots, z_{n}\right]$ by $p_{T}:=\prod_{i \rightarrow j}\left(z_{i}-z_{j}\right)$. Let $S_{n}$ act on $\mathbb{C}\left[z_{1}, \ldots, z_{n}\right]$ and its quotient field $\mathbb{C}\left(z_{1}, \ldots, z_{n}\right)$ in the obvious way, so that $w \cdot z_{i}=z_{w(i)}$.

\section{Proposition 2.5.}

(1) There is a linear map $\varrho: \mathcal{V}(1, n) \rightarrow \mathbb{C}\left(z_{1}, \ldots, z_{n}\right)$ such that $\varrho([T])=\frac{1}{p_{T}}, \forall T \in$ $\mathcal{T}(1, n)$.

(2) The map $\varrho$ is injective and $S_{n}$-equivariant.

(3) $\mathcal{V}(1, n)$ has basis $\left\{[T] \mid T \in \mathcal{T}(1, n)^{\circ}\right\}$.

Proof: To prove (1), it suffices to show that $\frac{1}{p_{T_{1}}}+\frac{1}{p_{T_{2}}}=\frac{1}{p_{T_{3}}}$ for $T_{1}, T_{2}, T_{3}$ a triple as in (2.2), and $\frac{1}{p_{T}}+\frac{1}{p_{T^{\prime}}}=0$ for $T, T^{\prime}$ as in (2.3); these are both trivial. The equivariance part of (2) is also obvious. Now by Lemma 2.4 , the set in (3) is a spanning set, so (2) and (3) will both follow from the statement that $\left\{\frac{1}{p_{T}} \mid T \in \mathcal{T}(1, n)^{\circ}\right\}$ is a linearly independent subset of $\mathbb{C}\left(z_{1}, \ldots, z_{n}\right)$. We could deduce this from Orlik-Solomon theory using (2.8) below, but there is also a pleasant direct proof by induction on $n$. The claim is vacuously true when $n=1$, so suppose that $n \geq 2$ and that

$$
\sum_{T \in \mathcal{T}(1, n)^{\circ}} \frac{c_{T}}{p_{T}}=0,
$$

for some $c_{T} \in \mathbb{C}$. Any $T \in \mathcal{T}(1, n)^{\circ}$ contains the edge $n-1 \rightarrow n$, and hence determines a partition $\{1, \ldots, n\}=A_{T} \sqcup B_{T}$, where $A_{T}$ consists of all the vertices of $T$ 
closer to $n-1$ than to $n$, and $B_{T}$ consists of all the vertices closer to $n$. Fix a partition $\{1, \ldots, n\}=A \sqcup B$, where $n-1 \in A$ and $n \in B$, and define

$$
\mathcal{T}(1, n)_{A, B}^{\circ}=\left\{T \in \mathcal{T}(1, n)^{\circ} \mid A_{T}=A, B_{T}=B\right\}
$$

It suffices to show that $c_{T}=0$ for all $T \in \mathcal{T}(1, n)_{A, B}^{\circ}$. Now we can rewrite (2.4) as

$$
\sum_{T \in \mathcal{T}(1, n)_{A, B}^{\circ}} c_{T} \prod_{\substack{i \rightarrow j \\ i \leq n-2}}\left(z_{i}-z_{j}\right)^{-1}+\sum_{\substack{T \in \mathcal{T}(1, n)^{\circ} \\ T \notin \mathcal{T}(1, n)_{A, B}^{\circ}}} c_{T} \prod_{\substack{i \rightarrow j \\ i \leq n-2}}\left(z_{i}-z_{j}\right)^{-1}=0
$$

where we have multiplied through by the common factor $z_{n-1}-z_{n}$. The terms in the first sum contain only factors $\left(z_{i}-z_{j}\right)^{-1}$ where $i, j$ are both in $A$ or both in $B$, whereas each of the terms in the second sum contains at least one factor $\left(z_{i}-z_{j}\right)^{-1}$ where one of $i, j$ is in $A$ and the other is in $B$. Therefore the first sum is itself zero: one way to see this is to make a substitution of variables $z_{a} \mapsto z_{a}+s$ for all $a \in A$ and $z_{b} \mapsto z_{b}+t$ for all $b \in B$, and then send $|s-t| \rightarrow \infty$, killing all terms in the second sum while leaving the first unchanged. We are now reduced to showing that the set

$$
\left\{\prod_{\substack{i \rightarrow j \\ T \\ i \leq n-2}}\left(z_{i}-z_{j}\right)^{-1} \mid T \in \mathcal{T}(1, n)_{A, B}^{\circ}\right\}
$$

is linearly independent. But a tree $T \in \mathcal{T}(1, n)_{A, B}^{\circ}$ is constructed from uniquely defined smaller trees $\left.T\right|_{A} \in \mathcal{T}(1, A)^{\circ}$ and $\left.T\right|_{B} \in \mathcal{T}(1, B)^{\circ}$, by adding the edge from $n-1$ to $n$ : so the set (2.6) is exactly

$$
\left\{\frac{1}{p_{T^{\prime}} p_{T^{\prime \prime}}} \mid T^{\prime} \in \mathcal{T}(1, A)^{\circ}, T^{\prime \prime} \in \mathcal{T}(1, B)^{\circ}\right\} .
$$

By the induction hypothesis, $\left\{\frac{1}{p_{T^{\prime}}} \mid T^{\prime} \in \mathcal{T}(1, A)^{\circ}\right\}$ is a linearly independent set in $\mathbb{C}\left(z_{a} \mid a \in A\right)$, and $\left\{\frac{1}{p_{T^{\prime \prime}}} \mid T^{\prime \prime} \in \mathcal{T}(1, B)^{\circ}\right\}$ is a linearly independent set in $\mathbb{C}\left(z_{b} \mid b \in B\right)$, so the claim follows.

We can now deduce the analogous basis for $\mathcal{V}(r, n)$ for general $r$.

Proposition 2.6. Define $Z:=\left\{\underline{\zeta}=\left(\zeta_{1}, \ldots, \zeta_{n}\right) \in \mu_{r}^{n} \mid \zeta_{n}=1\right\}$.

(1) $\mathcal{V}(1, n)$ can be identified with the subspace of $\mathcal{V}(r, n)$ spanned by $[T]$ for $T \in$ $\mathcal{T}(1, n)$.

(2) $\mathcal{V}(r, n)=\bigoplus_{\underline{\zeta} \in Z} \underline{\zeta} \cdot \mathcal{V}(1, n)$

(3) $\mathcal{V}(r, n)$ has basis $\left\{[T] \mid T \in \mathcal{T}(r, n)^{\circ}\right\}$.

(4) $\operatorname{dim} \mathcal{V}(r, n)=r^{n-1}(n-1)$ !.

Springer 
(5) Embed $\mu_{r} \times S_{n}$ in $W(r, n)$ by the map $(\zeta, w) \mapsto(\zeta, \ldots, \zeta) w$. As a representation of $W(r, n)$,

$$
\mathcal{V}(r, n) \cong \operatorname{Ind}_{\mu_{r} \times S_{n}}^{W(r, n)}(\mathcal{V}(1, n)),
$$

where $\mu_{r}$ acts trivially on $\mathcal{V}(1, n)$.

Proof: Identifying $\mathcal{T}(1, n)$ with the subset of $\mathcal{T}(r, n)$ where all the edge-labels are 1 , we have that for $\zeta \in Z$,

$$
\underline{\zeta} \cdot \mathcal{T}(1, n)=\left\{T \in \mathcal{T}(r, n) \mid i \stackrel{\eta}{\rightarrow} j \Longrightarrow \eta=\zeta_{i} \zeta_{j}^{-1}\right\}
$$

It is clear that for any tree $T \in \mathcal{T}(r, n)$, the edge labels satisfy this rule for a unique $\underline{\zeta} \in Z$, so $\mathcal{T}(r, n)$ is the disjoint union of these subsets $\underline{\zeta} \cdot \mathcal{T}(1, n)$. Moreover, the relations involve only trees in the same subset, which proves (1) and (2). Parts (3) and (4) follow from (2) and Proposition 2.5. Part (5) follows from (2) and the fact that $Z$ is a set of coset representatives for $W(r, n) /\left(\mu_{r} \times S_{n}\right)$.

There is another easy-to-describe basis of $\mathcal{V}(r, n)$. Let $T_{0} \in \mathcal{T}(1, n)$ be the tree (a chain) in which $i \rightarrow i+1$ for all $i \leq n-1$. For $T \in \mathcal{T}(r, n), \zeta \in Z$ and $w \in S_{n}$, we say that $(\zeta, w)$ refines $T$ if $i \stackrel{\eta}{\rightarrow} j$ in $T$ implies $w^{-1}(i)<w^{-\Gamma}(j)$ and $\eta=\zeta_{i} \zeta_{j}^{-1}$; in other words, $T \in \zeta . \mathcal{T}(1, n)$ and the total order on $\{1, \ldots, n\}$ determined by the chain $w \cdot T_{0}$ refines the partial order determined by $T$.

Proposition 2.7. For all $T \in \mathcal{T}(r, n)$, the equation

$$
[T]=\sum_{\substack{(\underline{\zeta}, w) \in Z \times S_{n} \\(\underline{\zeta}, w) \text { refines } T}}\left[\underline{\zeta} w \cdot T_{0}\right]
$$

holds in $\mathcal{V}(r, n)$.

Proof: Let $f(T)$ denote the right-hand side. If $T_{1}, T_{2}, T_{3} \in \mathcal{T}(r, n)$ are as in (2.2), it is clear that the set of $(\zeta, w)$ which refine $T_{3}$ is the disjoint union of the corresponding sets for $T_{1}$ and $T_{2}$, and hence $f\left(T_{3}\right)=f\left(T_{1}\right)+f\left(T_{2}\right)$. Now certainly $[T]=f(T)$ if $T$ is a chain, i.e. $T=\underline{\zeta} w \cdot T_{0}$ for some $(\underline{\zeta}, w)$. If $T$ is not of this form, then it forms part of a triple $\left(T_{1}, T_{2}, T\right)$ as in (2.2), where $T_{1}$ and $T_{2}$ strictly precede $T$ in the lexicographic ordering according to the $n$-tuple $\left(v_{0}, \ldots, v_{n-1}\right)$, where $v_{d}$ is the number of vertices at distance $d$ from the root. The result follows by induction.

Corollary 2.8. $\mathcal{V}(r, n)$ has basis $\left\{\left[\underline{\zeta} w . T_{0}\right] \mid \underline{\zeta} \in Z, w \in S_{n-1}\right\}$.

Proof: If $T \in \mathcal{T}(r, n)^{\circ}$, the partial order corresponding to $T$ has unique maximal element $n$, so if $(\underline{\zeta}, w)$ refines $T, w$ must lie in $S_{n-1}$. So (3) of Proposition 2.6 and 
Proposition 2.7 imply that the stated set spans $\mathcal{V}(r, n)$; it must be a basis by (4) of Proposition 2.6.

These results offer an alternative way of defining $\mathcal{V}(r, n)$. Consider the quotient $\widetilde{\mathcal{V}}(r, n)$ of $\mathbb{C} \mathcal{T}(r, n)$ obtained by imposing only the relations (2.2), and not (2.3). The above arguments show that the images of the chains in $\mathcal{T}(r, n)$ form a basis of $\widetilde{\mathcal{V}}(r, n)$. We can then regard $\mathcal{V}(r, n)$ as the quotient of the formal span of these chains by the relations (2.3), where $[T]$ and $\left[T^{\prime}\right]$ are interpreted by means of Proposition 2.7. Another way of saying this is that we can write explicit generators for the left ideal $I$ of $\mathbb{C} W(r, n)$ defined by $I=\left\{x \in \mathbb{C} W(r, n) \mid x .\left[T_{0}\right]=0\right\}$ : namely,

$$
(\zeta, \ldots, \zeta)-1, \text { for } \zeta \in \mu_{r}, \text { and } \sum_{w \in\left(S_{d} \times S_{n-d}\right) \backslash S_{n}} w, \quad \text { for } 1 \leq d \leq n-1 \text {. }
$$

(Here $\left(S_{d} \times S_{n-d}\right) \backslash S_{n}$ denotes the set of minimal-length left coset representatives for the Young subgroup $S_{d} \times S_{n-d}$.) Then $x \mapsto x$.[ $\left[T_{0}\right]$ gives an isomorphism $\mathbb{C} W(r, n) / I \stackrel{\sim}{\rightarrow} \mathcal{V}(r, n)$ of representations of $W(r, n)$. This point of view will not be used in the remainder of the paper.

Lehrer and Solomon have effectively already studied the representation $\mathcal{V}(1, n)$. Indeed, in [11, Section 3] they consider the top-degree component $A^{n-1}(M(n))$ of the Orlik-Solomon algebra of the hyperplane complement

$$
M(n):=\left\{\left(z_{1}, \ldots, z_{n}\right) \in \mathbb{C}^{n} \mid z_{i} \neq z_{j}, \forall i \neq j\right\}
$$

This component has a 'no-broken-circuit' basis $\left\{\alpha_{T} \mid T \in \mathcal{T}(1, n)^{\circ}\right\}$ where $\alpha_{T}:=\bigwedge_{i \rightarrow j}\left(\frac{d z_{i}-d z_{j}}{z_{i}-z_{j}}\right)$, with the factors of the wedge product ordered by increasing $i$. But by an elementary calculation,

$$
\alpha_{T}=\frac{1}{p_{T}} \alpha, \text { where } \alpha:=\sum_{i=1}^{n}(-1)^{n-i} d z_{1} \wedge \cdots \wedge \widehat{d z_{i}} \wedge \cdots \wedge d z_{n}
$$

(The hat denotes omission of that factor.) So we have a linear isomorphism $\mathcal{V}(1, n) \stackrel{\sim}{\rightarrow}$ $A^{n-1}(M(n)): x \mapsto \varrho(x) \alpha$, and since $w . \alpha=\varepsilon_{n}(w) \alpha$ for all $w \in S_{n}$, this gives an isomorphism

$$
\varepsilon_{n} \otimes \mathcal{V}(1, n) \cong A^{n-1}(M(n))
$$

of representations of $S_{n}$. Under this isomorphism, the $r=1$ case of Corollary 2.8 corresponds to [11, Proposition (3.3)], and the main result [11, Theorem (3.9)] becomes the following fact about $\mathcal{V}(1, n)$. Let $\psi_{n}: \mu_{n} \hookrightarrow \mathbb{C}^{\times}$be the inclusion map, and simultaneously identify $\mu_{n}$ with a subgroup of $S_{n}$, by sending a generator $\zeta_{0} \in \mu_{n}$ to the $n$-cycle $(1,2, \ldots, n)$.

Theorem 2.9. As a representation of $S_{n}, \mathcal{V}(1, n) \cong \operatorname{Ind}_{\mu_{n}}^{S_{n}}\left(\psi_{n}\right)$. 
Proof: To emphasize that Lehrer's and Solomon's proof is purely combinatorial, we will sketch its translation into the context of $\mathcal{V}(1, n)$. Since $\operatorname{dim} \mathcal{V}(1, n)=(n-$ $1) !=\left|S_{n} / \mu_{n}\right|$, it suffices to show that $\mathcal{V}(1, n)$ is generated as a $\mathbb{C} S_{n}$-module by some $x$ satisfying $\theta \cdot x=\psi_{n}(\theta) x$ for all $\theta \in \mu_{n}$. An element which indeed has the latter property is $x=\left(\sum_{p=0}^{n-1} \zeta_{0}^{-p}(1,2, \ldots, n)^{p}\right)$. [ $\left.T_{0}\right]$. The claim is that

$$
x=\left(1-c_{n-1} \zeta_{0}^{-1}\right)\left(1-c_{n-2} \zeta_{0}^{-1}\right) \cdots\left(1-c_{2} \zeta_{0}^{-1}\right)\left(1-c_{1} \zeta_{0}^{-1}\right) \cdot\left[T_{0}\right]
$$

where $c_{i}$ is the $i$-cycle $(1,2, \ldots, i)$. Since each $1-c_{i} \zeta_{0}^{-1}$ for $i \leq n-1$ is an invertible element of $\mathbb{C} S_{n}$, and $\mathcal{V}(1, n)$ is certainly generated as a $\mathbb{C} S_{n}$-module by $\left[T_{0}\right]$ (by Corollary 2.8), this finishes the proof. To prove the claim (2.10), we define elements $b_{n, p} \in \mathbb{C} S_{n-1}$ by the rule

$$
\left(1-c_{n-1} t\right)\left(1-c_{n-2} t\right) \cdots\left(1-c_{2} t\right)\left(1-c_{1} t\right)=\sum_{p=0}^{n-1} b_{n, p} t^{p}
$$

It suffices to show that $c_{n}^{p} \cdot\left[T_{0}\right]=b_{n, p} .\left[T_{0}\right]$, for all $0 \leq p \leq n-1$. The $p=0$ case is trivial, and the $p=n-1$ case is equivalent to $w_{0} \cdot\left[T_{0}\right]=(-1)^{n-1}\left[T_{0}\right]$ where $w_{0}$ is the permutation $i \mapsto n+1-i$, which is an immediate consequence of (2.3). For the remaining cases $1 \leq p \leq n-2$ we use induction on $n$, and the obvious recurrence $b_{n, p}=b_{n-1, p}-c_{n-1} b_{n-1, p-1}$. By the induction hypothesis, we have

$$
b_{n-1, p} \cdot[1 \rightarrow 2 \rightarrow \cdots \rightarrow n-1]=[p+1 \rightarrow \cdots \rightarrow n-1 \rightarrow 1 \rightarrow \cdots \rightarrow p],
$$

which, bearing in mind $b_{n-1, p} \in \mathbb{C} S_{n-2}$, implies that

$$
\begin{aligned}
b_{n-1, p \cdot\left[T_{0}\right]} & =-b_{n-1, p} \cdot\left[\begin{array}{cc}
1 \rightarrow 2 \rightarrow \cdots \rightarrow n-1 \\
\uparrow \\
n
\end{array}\right] \\
& =-\left[\begin{array}{c}
p+1 \rightarrow \cdots \rightarrow \\
\uparrow-1 \rightarrow 1 \rightarrow \cdots \rightarrow p \\
n
\end{array}\right] .
\end{aligned}
$$

Hence also

$$
c_{n-1} b_{n-1, p-1} .\left[T_{0}\right]=-\left[\begin{array}{c}
p+1 \rightarrow \cdots \rightarrow n-1 \rightarrow \cdots \rightarrow p \\
n \\
n
\end{array}\right] .
$$

The desired equation $c_{n}^{p} .\left[T_{0}\right]=b_{n-1, p} .\left[T_{0}\right]-c_{n-1} b_{n-1, p-1} .\left[T_{0}\right]$ is now a case of (2.2).

Combining our embeddings $\mu_{n} \hookrightarrow S_{n}$ and $\mu_{r} \times S_{n} \hookrightarrow W(r, n)$, we can embed $\mu_{r} \times$ $\mu_{n}$ in $W(r, n)$. 
Corollary 2.10. As a representation of $W(r, n)$,

$$
\mathcal{V}(r, n) \cong \operatorname{Ind}_{\mu_{r} \times \mu_{n}}^{W(r, n)}\left(1 \times \psi_{n}\right)
$$

Proof: This follows from (5) of Proposition 2.6 and Theorem 2.9.

We will need a generalization of Theorem 2.9. Suppose for the remainder of this section that $r \mid n$, and embed $W(r, n / r)$ in $S_{n}$ as the centralizer of the product of $n / r$ disjoint $r$-cycles. Hence $\mu_{r} \times \mu_{n / r}$ is also embedded in $S_{n}$.

Theorem 2.11. As a representation of $S_{n}$,

$$
\mathcal{V}(1, n) \cong \operatorname{Ind}_{\mu_{r} \times \mu_{n / r}}^{S_{n}}\left(\psi_{r}^{n / r} \times \psi_{n / r}\right) \cong \operatorname{Ind}_{W(r, n / r)}^{S_{n}}\left(\operatorname{prod}_{n / r} \otimes \mathcal{V}(r, n / r)\right) .
$$

Proof: The second isomorphism follows from Corollary 2.10. It would be good to realize the first isomorphism explicitly, as we did in the $r=1$ case (Theorem 2.9), by finding a generator $x$ for $\mathcal{V}(1, n)$ which satisfies $\zeta . x=\psi_{r}(\zeta)^{n / r} x$ for all $\zeta \in \mu_{r}$ and $\theta \cdot x=\psi_{n / r}(\theta)$ for all $\theta \in \mu_{n / r}$. However, I have not been able to do this. We will instead prove that the characters coincide:

$$
\chi\left(\operatorname{Ind}_{\mu_{n}}^{S_{n}}\left(\psi_{n}\right)\right)=\chi\left(\operatorname{Ind}_{\mu_{r} \times \mu_{n / r}}^{S_{n}}\left(\psi_{r}^{n / r} \times \psi_{n / r}\right)\right) .
$$

Both sides are clearly supported on the elements of cycle type $\left(d^{n / d}\right)$ where $d \mid n$. The value of the left-hand side at such an element, when multiplied by the index of the centralizer, is $\sum_{\zeta \in \mu_{d}^{\circ}} \zeta=\mu(d)$, where $\mu_{d}^{\circ}$ denotes the set of primitive $d$ th roots of 1 , and $\mu(d)$ is the Möbius $\mu$-function. The value of the right-hand side at such an element, when multiplied by the index of the centralizer, is

$$
\begin{aligned}
\sum_{\substack{e|r \\
f|(n / r) \\
\operatorname{lcm}(e, f)=d}} \sum_{\substack{\eta \in \mu_{e}^{\circ} \\
\theta \in \mu_{f}^{\circ}}} \eta^{n / r} \theta & =\sum_{\substack{e|r \\
f|(n / r) \\
\operatorname{ccm}(e, f)=d}} \mu(f) \sum_{\eta \in \mu_{e}^{\circ}} \eta^{n / r} \\
& =\sum_{\substack{e|r \\
f|(n / r) \\
\operatorname{lcm}(e, f)=d}} \frac{\mu(f) \mu\left(\frac{e}{\operatorname{gcd}(n / r, e)}\right) \phi(e)}{\phi\left(\frac{e}{\operatorname{gcd}(n / r, e)}\right)},
\end{aligned}
$$

where $\phi$ is Euler's function. Since both formulas are multiplicative, it suffices to prove that they are equal when $r=p^{a}, n / r=p^{b}, d=p^{c}$ for some prime $p$ and $a, b, c \in \mathbb{N}$, $c \leq a+b$. That is, we must prove

$$
\mu\left(p^{c}\right)=\sum_{\substack{0 \leq x \leq a \\ 0 \leq y \leq b \\ \max \{x, y\}=c}} \frac{\mu\left(p^{y}\right) \mu\left(p^{x-\min \{b, x\}}\right) \phi\left(p^{x}\right)}{\phi\left(p^{x-\min \{b, x\}}\right)} .
$$


If $c=0$, both sides are clearly 1 . If $c=1$ and $a=0$, the only term on the right-hand side is $x=0, y=1$, and both sides are -1 . If $c=1$ and $b=0$, the only term on the right-hand side is $x=1, y=0$, and both sides are again -1 . If $c=1$ and $a, b \geq 1$, there are three terms on the right-hand side, those where $x, y \in\{0,1\}$ are not both 0 ; their sum is once again -1 . So we may assume that $c \geq 2$, in which case the left-hand side is 0 . Because of the $\mu$-function factors, the only nonzero terms on the right-hand side are those where $y \leq 1$ and $x \leq b+1$. The first of these conditions forces $x=c$, so if $c>b+1$ or $c>a$ there are no nonzero terms at all, and we are done. The only remaining case is that $c \geq 2, c \leq a, c \leq b+1$, in which case the two nonzero terms $x=c, y=0$ and $x=c, y=1$ cancel each other out.

\section{Cohomology of the hyperplane complement $T(r, n)$}

As in the previous section, $r$ and $n$ denote positive integers. Define

$$
T(r, n):=\left\{\left(z_{1}, z_{2}, \ldots, z_{n}\right) \in \mathbb{C}^{n} \mid z_{i} \neq 0, \forall i, z_{i}^{r} \neq z_{j}^{r}, \forall i \neq j\right\}
$$

This is the complement of the hyperplanes $z_{i}=0$ for $1 \leq i \leq n$ and $z_{i}=\eta z_{j}$ for $1 \leq i \neq j \leq n, \eta \in \mu_{r}$. The wreath product $W(r, n)$ acts on $T(r, n)$ by the restriction of its standard representation on $\mathbb{C}^{n}$. Indeed, if $r \geq 2$, the hyperplanes we have removed are exactly the reflecting hyperplanes for the image $G(r, 1, n)$ of this representation; if $r=1$, we have also removed the hyperplanes $z_{i}=0$.

Let $\Omega^{\bullet}(T(r, n))$ be the graded anti-commutative algebra of regular differential forms on $T(r, n)$. Thus $\Omega^{0}(T(r, n))=\mathcal{O}(T(r, n))$ is the algebra of regular functions on $T(r, n)$, or in other words the subring $\mathbb{C}\left[z_{i}^{ \pm 1},\left(z_{i}-\eta z_{j}\right)^{-1}\right]$ of $\mathbb{C}\left(z_{1}, \ldots, z_{n}\right)$, and for $1 \leq p \leq n, \Omega^{p}(T(r, n))$ is the free $\Omega^{0}(T(r, n))$-module with basis $d z_{i_{1}} \wedge \cdots \wedge$ $d z_{i_{p}}$, for $1 \leq i_{1}<\cdots<i_{p} \leq n$. We have the usual differential $d: \Omega^{\bullet}(T(r, n)) \rightarrow$ $\Omega^{\bullet}(T(r, n))$ raising degrees by 1 . As with any nonsingular affine complex variety, the cohomology of the cochain complex $\left(\Omega^{\bullet}(T(r, n)), d\right)$ is merely the cohomology $H^{p}(T(r, n), \mathbb{C})$. The natural action of $W(r, n)$ on $\Omega^{\bullet}(T(r, n))$ preserves the grading, the wedge product, and the differential. Explicitly, the action on $\Omega^{0}(T(r, n))$ is given by the formulas:

$$
\begin{aligned}
& \underline{\zeta} \cdot f\left(z_{1}, \ldots, z_{n}\right)=f\left(\zeta_{1}^{-1} z_{1}, \ldots, \zeta_{n}^{-1} z_{n}\right) \\
& w \cdot f\left(z_{1}, \ldots, z_{n}\right)=f\left(z_{w(1)}, \ldots, z_{w(n)}\right)
\end{aligned}
$$

and the action on $\Omega^{p}(T(r, n))$ uses the additional rule $w \cdot d z_{i}=d z_{w(i)}$.

As mentioned in the introduction, the cohomology of a hyperplane complement can be described quite explicitly by results of Brieskorn, Orlik and Solomon; in our case, this description is as follows. Let $A^{\bullet}(T(r, n))$ be the subalgebra of $\Omega^{\bullet}(T(r, n))$ generated by the following 1-forms:

$$
\omega_{i}:=\frac{d z_{i}}{z_{i}}, \forall i, \quad \text { and } \quad \omega_{i, j, \eta}:=\frac{d z_{i}-\eta d z_{j}}{z_{i}-\eta z_{j}}, \quad \forall i \neq j, \eta \in \mu_{r} .
$$


Since these generators are closed, $d \alpha=0$ for all $\alpha \in A^{\bullet}(T(r, n))$.

Theorem 3.1. (Brieskorn, see [13, Theorem 5.89]). The inclusion $\left(A^{\bullet}(T(r, n)), 0\right) \hookrightarrow$ $\left(\Omega^{\bullet}(T(r, n)), d\right)$ of cochain complexes is a quasi-isomorphism. In other words, $A^{p}(T(r, n)) \cong H^{p}(T(r, n), \mathbb{C})$ via the map sending a form $\alpha$ to its cohomology class.

Theorem 3.2. (Orlik-Solomon, see [13, Theorems 3.43 and 3.126]). $A^{\bullet}(T(r, n))$ has a basis consisting of all products $\alpha_{1} \wedge \alpha_{2} \wedge \cdots \wedge \alpha_{n}$, where each $\alpha_{i}$ is either $1, \omega_{i}$, or $\omega_{i, j, \eta}$ for some $j>i$ and $\eta \in \mu_{r}$.

To obtain the basis given here as the 'no-broken-circuit' basis of [13, Theorem 3.43], order the hyperplanes so that the hyperplanes $\left\{z_{i}=\eta z_{j}\right\}$ for $j>i$ come first, in lexicographic order of $(i, j)$, and then the hyperplanes $\left\{z_{i}=0\right\}$, in order of $i$. If $r=1$, we recover the basis used by Lehrer in [8] (identify $A^{\bullet}(T(1, n)$ ) with the Orlik-Solomon algebra of $S_{n+1}$ by rewriting $\omega_{i}$ as $\left.\omega_{i, n+1}\right)$. If $r=2$, we recover the basis used by Lehrer in [9].

Of course, the basis in Theorem 3.2 is not $W(r, n)$-stable, so it is necessary to consider the linear relations satisfied by the set of all $W(r, n)$-translates of basis elements. This set can be parametrized by an enhanced version of the trees considered in the previous section.

Definition 3.3. A directed forest on $\{1, \ldots, n\}$ is a directed graph with vertex set $\{1, \ldots, n\}$ such that each connected component is a directed tree on the vertices it contains. A decorated directed forest $F$ on $\{1, \ldots, n\}$ is a directed forest on $\{1, \ldots, n\}$ equipped with, firstly, a labelling of each edge by an element of $\mu_{r}$, and, secondly, a partition of the set of roots into open and closed roots. We write $i \stackrel{\eta}{\rightarrow} j$ (with a subscript $F$ when necessary) to mean that there is an edge from $i$ to $j$ in $F$ whose label is $\eta \in \mu_{r}$. We say that $F$ is rectified if $i \stackrel{\zeta}{\rightarrow} j$ implies $i<j$. Let $\mathcal{F}(r, n)$ be the set of decorated directed forests on $\{1, \ldots, n\}$, and let $\mathcal{F}(r, n)^{k, l}$ be the subset of $\mathcal{F}(r, n)$ consisting of those forests with $k$ edges and $l$ closed roots (and hence $n-k-l$ open roots). Write $\mathcal{F}(r, n)^{\circ}$ and $\mathcal{F}(r, n)^{\circ, k, l}$ for the rectified subsets of these.

We define an action of $W(r, n)$ on $\mathcal{F}(r, n)$ by the same rules as for $\mathcal{T}(r, n)$. (As well as permuting the vertices, $S_{n}$ respects the openness of roots; in other words, $i$ is an open root of $F$ if and only if $w(i)$ is an open root of $w . F$.) Clearly each $\mathcal{F}(r, n)^{k, l}$ is a $W(r, n)$-stable subset.

We can now define a suitably signed version of the set of $W(r, n)$-translates of the Orlik-Solomon basis elements.

Definition 3.4. For any decorated directed forest $F \in \mathcal{F}(r, n)^{k, l}$, and any $i \in$ $\{1, \ldots, n\}$, define the sign

$$
\varepsilon_{i}(F):=(-1)^{i-1-\mid\{\text { open roots of } F \text { which are }<i\} \mid} .
$$


Define $\alpha(F) \in A^{k+l}(T(r, n))$ by $\alpha_{1}(F) \wedge \alpha_{2}(F) \wedge \cdots \wedge \alpha_{n}(F)$, where

$$
\alpha_{i}(F):= \begin{cases}\varepsilon_{i}(F), & \text { if } i \text { is an open root of } F, \\ \omega_{i}, & \text { if } i \text { is a closed root of } F, \\ \omega_{i, j, \eta}, & \text { if } i \stackrel{\eta}{\rightarrow} j \text { in } F .\end{cases}
$$

Note that if $i$ is not an open root of $F, \varepsilon_{i}(F)$ is exactly the sign incurred in moving the $\alpha_{i}(F)$ factor of the wedge product to the front.

Since the wedge products in Theorem 3.2 are, up to sign, those $\alpha(F)$ for $F$ rectified, we can rewrite that result as follows.

Theorem 3.5. $A^{p}(T(r, n))$ has basis $\left\{\alpha(F) \mid F \in \bigcup_{k+l=p} \mathcal{F}(r, n)^{\circ, k, l}\right\}$.

Example 3.6. The twelve elements of $\mathcal{F}(2,2)$, with the corresponding elements of $A^{\bullet}(2,2)$, are as follows; asterisks indicate closed roots.

(Note that $2 \stackrel{ \pm 1}{\longrightarrow} 1$ and $2 \stackrel{ \pm 1}{\longrightarrow} 1^{*}$ are not rectified.)

\begin{tabular}{lrc}
\hline 1 & 2 & 1 \\
$1 \stackrel{ \pm 1}{\longrightarrow} 2$ & $-\omega_{1,2, \pm 1}$ \\
$2 \stackrel{ \pm 1}{\longrightarrow} 1$ & $\omega_{2,1, \pm 1}$ \\
1 & $2^{*}$ & $\omega_{2}$ \\
$1^{*} \quad 2$ & $-\omega_{1}$ \\
$1 \stackrel{ \pm 1}{\longrightarrow} 2^{*}$ & $\omega_{1,2, \pm 1} \wedge \omega_{2}$ \\
$2 \stackrel{ \pm 1}{\longrightarrow} 1^{*}$ & $\omega_{1} \wedge \omega_{2,1, \pm 1}$ \\
$1^{*} 2^{*}$ & $\omega_{1} \wedge \omega_{2}$ \\
& &
\end{tabular}

The justification for the above definition of $\alpha(F)$ is the following result, in which the $\operatorname{sign} \varepsilon(w, F)$ is defined by

$$
\varepsilon(w, F):=(-1)^{\mid\{(i, j) \mid i, j \text { open roots of } F, i<j, w(i)>w(j)\} \mid} .
$$

Proposition 3.7. For $\underline{\zeta} \in \mu_{r}^{n}, w \in S_{n}$ and $F \in \mathcal{F}(r, n)$,

$$
\alpha(\underline{\zeta} w \cdot F)=\varepsilon_{n}(w) \varepsilon(w, F) \underline{\zeta} w \cdot \alpha(F) .
$$

Proof: That $\underline{\zeta} . \alpha(F)=\alpha(\underline{\zeta} . F)$ is obvious from the fact that $\underline{\zeta} . \omega_{i, j, \eta}=\omega_{i, j, \eta \zeta_{i}} \zeta_{j}^{-1}$. So we need only show that $\alpha(w . F)=\varepsilon_{n}(w) \varepsilon(w, F) w . \alpha(F)$, which it suffices to prove in the case that $w$ is a simple transposition $s_{i}=(i, i+1)$, since $\varepsilon\left(w w^{\prime}, F\right)=$ $\varepsilon\left(w, w^{\prime} F\right) \varepsilon\left(w^{\prime}, F\right)$. It is clear that $\alpha_{j}\left(s_{i} . F\right)=s_{i} \cdot \alpha_{j}(F)$ for all $j \neq i, i+1$, so we are 
reduced to showing

$$
\alpha_{i}\left(s_{i} . F\right) \wedge \alpha_{i+1}\left(s_{i} . F\right)=\left\{\begin{array}{lc}
s_{i} \cdot \alpha_{i}(F) \wedge s_{i} \cdot \alpha_{i+1}(F), & \text { if } i, i+1 \text { are open } \\
-s_{i} \cdot \alpha_{i}(F) \wedge s_{i} \cdot \alpha_{i+1}(F), & \text { otherwise }
\end{array}\right.
$$

Now if $i$ and $i+1$ are open roots of $F$, both sides are clearly 1 . If neither $i$ nor $i+1$ is an open root of $F$, then $\alpha_{i}\left(s_{i} . F\right)=s_{i} . \alpha_{i+1}(F)$ and $\alpha_{i+1}\left(s_{i} . F\right)=s_{i} . \alpha_{i}(F)$, both being 1-forms, so the claim holds. If $i+1$ is an open root of $F$ and $i$ is not, then $\alpha_{i+1}\left(s_{i} . F\right)=s_{i} \cdot \alpha_{i}(F)$ but

$$
\begin{aligned}
\varepsilon_{i}\left(s_{i} . F\right) & =(-1)^{\left.i-1-\mid \text { open roots of } s_{i} . F \text { which are }<i\right\} \mid} \\
& =(-1)^{i-1-\mid\{\text { open roots of } F \text { which are }<i+1\} \mid}=-\varepsilon_{i+1}(F),
\end{aligned}
$$

so again the claim holds. The case where $i$ is an open root of $F$ and $i+1$ is not is similar.

We now describe the linear relations satisfied by the elements $\alpha(F)$, when $F$ is not necessarily rectified; these were the motivation for the relations used in the previous section.

Proposition 3.8. The following hold in $A^{\bullet}(T(r, n))$.

(1) $\alpha\left(F_{1}\right)+\alpha\left(F_{2}\right)=\alpha\left(F_{3}\right)$, if $F_{1}, F_{2}, F_{3} \in \mathcal{F}(r, n)$ are identical except for the edges between three vertices $i, j$, and $k$, where we have

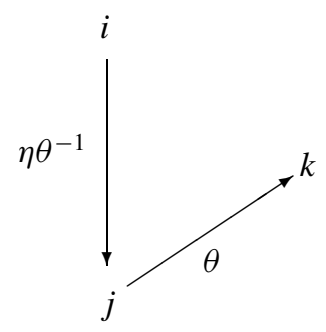

$F_{1}$

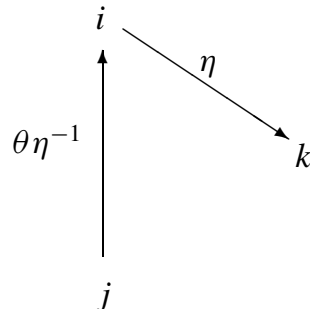

$F_{2}$

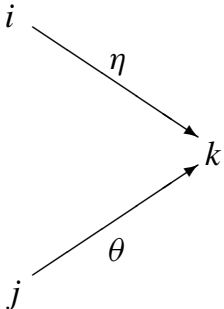

$F_{3}$

for some $\eta, \theta \in \mu_{r}$. (The assertion that they are otherwise identical includes the fact that if $k$ is a root of any, hence all, of these forests, it is either open in all or closed in all.)

(2) $\alpha(F)+\alpha\left(F^{\prime}\right)=0$, if $F, F^{\prime} \in \mathcal{T}(r, n)$ are identical except that $j$ is an open root of $F, i$ is an open root of $F^{\prime}$, and we have $i \stackrel{\eta}{\rightarrow} j$ in $F, j \stackrel{\eta^{1}}{\rightarrow} i$ in $F^{\prime}$ for some $\eta \in \mu_{r}$.

(3) $\alpha(F)+\alpha\left(F^{\prime}\right)=\alpha\left(F^{\prime \prime}\right)$, if $F, F^{\prime}, F^{\prime \prime} \in \mathcal{F}(r, n)$ are identical except that $j$ is a closed root of $F$, $i$ is a closed root of $F^{\prime}$, both are closed roots of $F^{\prime \prime}$, and we have $i \stackrel{\eta}{\rightarrow} j$ in $F, j \stackrel{\eta^{\prime}}{\rightarrow} i$ in $F^{\prime}$ for some $\eta \in \mu_{r}$ (there is of course no edge between $i$ and $j$ in $F^{\prime \prime}$ ). 
Proof: In (1) and (3), the factors in various positions of the wedge product are forms of the same degrees in all three terms, so we may reorder the factors in the same way in all three terms so as to bring the factors in which the terms differ to the front, and then neglect the other factors. Therefore to prove these relations we need only check:

$$
\begin{gathered}
\omega_{i, j, \eta \theta^{-1}} \wedge \omega_{j, k, \theta}+\omega_{i, k, \eta} \wedge \omega_{j, i, \theta \eta^{-1}}=\omega_{i, k, \eta} \wedge \omega_{j, k, \theta}, \quad \text { and } \\
\omega_{i, j, \eta} \wedge \omega_{j}+\omega_{i} \wedge \omega_{j, i, \eta^{-1}}=\omega_{i} \wedge \omega_{j}
\end{gathered}
$$

These are known relations in $A^{\bullet}(T(r, n))$ (besides being trivial to verify directly). In (2), reorder the factors of the wedge products so that the factor corresponding to $i$ comes first, that corresponding to $j$ comes second, and the others (which are the same in both terms) follow in order. In the case of $\alpha(F)$, the sign incurred by this reordering is $\varepsilon_{i}(F)$, and the reordered product is $\omega_{i, j, \eta} \wedge \varepsilon_{j}(F) \wedge \cdots$. In the case of $\alpha\left(F^{\prime}\right)$, the sign incurred is $\varepsilon_{j}\left(F^{\prime}\right)$, and the reordered product is $\varepsilon_{i}\left(F^{\prime}\right) \wedge \omega_{j, i, \eta^{-1}} \wedge \cdots$. Since $\omega_{i, j, \eta}=\omega_{j, i, \eta^{-1}}$, we need only show that $\varepsilon_{i}(F) \varepsilon_{j}(F)+\varepsilon_{i}\left(F^{\prime}\right) \varepsilon_{j}\left(F^{\prime}\right)=0$. But if $i<j$, then $\varepsilon_{i}\left(F^{\prime}\right)=\varepsilon_{i}(F)$ while $\varepsilon_{j}\left(F^{\prime}\right)=-\varepsilon_{j}(F)$, because $i$ is an open root of $F^{\prime}$ but not of $F$; the $j<i$ case is similar.

From the viewpoint of the previous section, relation (3) of Proposition 3.8 appears to be of the wrong form. However, we can make $\alpha(F)+\alpha\left(F^{\prime}\right)$ zero as expected if we work modulo forests with a greater number of closed roots (such as $F^{\prime \prime}$ ). So we define for each $p$ a $W(r, n)$-stable filtration

$$
A^{p}(T(r, n))=A^{p}(T(r, n))_{0} \supseteq A^{p}(T(r, n))_{1} \supseteq A^{p}(T(r, n))_{2} \cdots,
$$

where

$$
A^{p}(T(r, n))_{m}:=\operatorname{span}\left\{\alpha(F) \mid F \in \bigcup_{\substack{k+l=p \\ l \geq m}} \mathcal{F}(r, n)^{k, l}\right\} .
$$

(In the $r=2$ case, a similar filtration was used in [3].) Also define $A^{k, l}(T(r, n)):=$ $A^{k+l}(T(r, n))_{l} / A^{k+l}(T(r, n))_{l+1}$, and for $F \in \mathcal{F}(r, n)^{k, l}$, let $[F]$ denote the image of $\alpha(F)$ in $A^{k, l}(T(r, n))$. We can now state the main result of this section.

Theorem 3.9. Let $r, n, p, k, l$ be as above.

(1) $A^{p}(T(r, n))$ can be defined abstractly as the vector space spanned by $\{\alpha(F) \mid F \in$ $\left.\bigcup_{k+l=p} \mathcal{F}(r, n)^{k, l}\right\}$ subject to the relations in Proposition 3.8.

(2) $A^{k, l}(T(r, n))$ can be defined abstractly as the vector space spanned by $\{[F] \mid F \in$ $\left.\mathcal{F}(r, n)^{k, l}\right\}$ subject to the following relations:

- $\left[F_{1}\right]+\left[F_{2}\right]=\left[F_{3}\right]$ whenever $F_{1}, F_{2}, F_{3} \in \mathcal{F}(r, n)^{k, l}$ are as in (1) of Proposition 3.8 ;

- $[F]+\left[F^{\prime}\right]=0$ whenever $F, F^{\prime} \in \mathcal{F}(r, n)^{k, l}$ are as in (2) or (3) of Proposition 3.8 .

(3) $A^{k, l}(T(r, n))$ has basis $\left\{[F] \mid F \in \mathcal{F}(r, n)^{\circ, k, l}\right\}$. 
(4) For $\underline{\zeta} \in \mu_{r}^{n}, w \in S_{n}$ and $F \in \mathcal{F}(r, n)^{k, l}$,

$$
\underline{\zeta} w \cdot[F]=\varepsilon_{n}(w) \varepsilon(w, F)[\underline{\zeta} w \cdot F]
$$

(5) As a representation of $W(r, n)$,

$$
A^{p}(T(r, n)) \cong \bigoplus_{k+l=p} A^{k, l}(T(r, n))
$$

Proof: The vector space defined abstractly as in (1) certainly maps to $A^{p}(T(r, n))$, since the stated relations do hold. So to prove (1), it suffices to show that the basis elements $\left\{\alpha(F) \mid F \in \bigcup_{k+l=p} \mathcal{F}(r, n)^{\circ, k, l}\right\}$ of Theorem 3.5 span the abstract vector space also. This follows from the same sort of rectification procedure as in Lemma 2.4: for $F \in \mathcal{F}(r, n)^{k, l}$, we use relations (2) and (3) of Proposition 3.8 to shift the root of each tree to its largest vertex, and any forests which arise from the right-hand side of relation (3) can be neglected, by downward induction on the number of closed roots. Once the root of each tree is its largest vertex, the rectification proceeds using relation (1) as in Lemma 2.4. So part (1) of the present result is proved. Moreover, this procedure expresses each $\alpha(F)$ as a linear combination of $\alpha\left(F^{\prime}\right)$ 's for $F^{\prime}$ rectified with at least as many closed roots as $F$. Therefore $A^{p}(T(r, n))_{m}$ has basis $\left\{\alpha(F) \mid F \in \bigcup_{k+l=p} \mathcal{F}(r, n)^{\circ}, k, l\right\}$, from which part (3) follows immediately. Part (2) follows from (3) by the same argument as for (1). Part (4) clearly follows from Proposition 3.7, and part (5) from complete reducibility of finite-dimensional representations of $W(r, n)$.

We can now relate $A^{k, l}(T(r, n))$ to the representations considered in the previous section. Recall that if $\lambda=\left(\lambda_{1}, \ldots, \lambda_{\ell}\right)$ is a partition in the usual combinatorial sense, the stabilizer in $S_{|\lambda|}$ of a set partition of $\{1, \ldots,|\lambda|\}$ into parts of sizes $\lambda_{1}, \ldots, \lambda_{\ell}$ is isomorphic to

$$
\left(S_{\lambda_{1}} \times \cdots \times S_{\lambda_{\ell}}\right) \rtimes\left(S_{m_{1}(\lambda)} \times S_{m_{2}(\lambda)} \times \cdots\right),
$$

where $S_{m_{i}(\lambda)}$ acts by permuting the $S_{\lambda_{a}}$ factors where $\lambda_{a}=i$. The subgroups of $W(r, n)$ referred to in the following result are defined similarly.

Corollary 3.10. As a representation of $W(r, n), \varepsilon_{n} \otimes A^{k, l}(T(r, n))$ is isomorphic to the following direct sum:

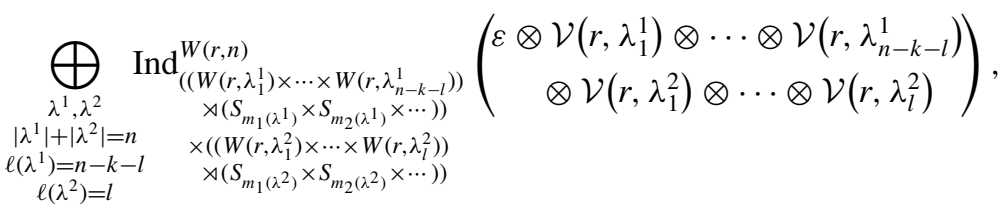


where $W\left(r, \lambda_{a}^{j}\right)$ acts on the $\mathcal{V}\left(r, \lambda_{a}^{j}\right)$ factor, $S_{m_{i}\left(\lambda^{j}\right)}$ acts by permuting the $\mathcal{V}\left(r, \lambda_{a}^{j}\right)$ factors where $\lambda_{a}^{j}=i$, and $\varepsilon$ denotes the product of the sign characters of the $S_{m_{i}\left(\lambda^{1}\right)}$ components.

Proof: For any partition of the set $\{1, \ldots, n\}$ into nonempty subsets $B_{1}, \ldots, B_{n-k-l}$, $C_{1}, \ldots, C_{l}$, let $\mathcal{F}(r, n)_{\left(\left\{B_{i}\right\},\left\{C_{j}\right\}\right)}^{k, l} \subset \mathcal{F}(r, n)^{k, l}$ be the subset consisting of forests in which the trees with open roots have vertex sets $B_{1}, \ldots, B_{n-k-l}$ and the trees with closed roots have vertex sets $C_{1}, \ldots, C_{l}$. Let $A^{k, l}(T(r, n))_{\left(\left\{B_{i}\right\},\left\{C_{j}\right\}\right)}$ be the subspace of $A^{k, l}(T(r, n))$ spanned by the elements $[F]$ for $F \in \mathcal{F}(r, n)_{\left(\left\{B_{i}\right\},\left\{C_{j}\right\}\right)}^{k, l}$. Since every relation in (2) of Theorem 3.9 involves forests in the same subset $\mathcal{F}(r, n)_{\left(\left\{B_{i}\right\},\left\{C_{j}\right\}\right)}^{k, l}$, we have a direct sum decomposition

$$
A^{k, l}(T(r, n))=\bigoplus_{\left(\left\{B_{i}\right\},\left\{C_{j}\right\}\right)} A^{k, l}(T(r, n))_{\left(\left\{B_{i}\right\},\left\{C_{j}\right\}\right)}
$$

Comparing the relations in Theorem 3.9 to those in the definition of $\mathcal{V}(r, n)$, we see that there is an isomorphism of vector spaces

$$
\begin{array}{r}
A^{k, l}(T(r, n))_{\left(\left\{B_{i}\right\},\left\{C_{j}\right\}\right)} \stackrel{\sim}{\rightarrow} \mathcal{V}\left(r, B_{1}\right) \otimes \cdots \otimes \mathcal{V}\left(r, B_{n-k-l}\right) \\
\otimes \mathcal{V}\left(r, C_{1}\right) \otimes \cdots \otimes \mathcal{V}\left(r, C_{l}\right)
\end{array}
$$

sending $[F]$ for $F \in \mathcal{F}(r, n)_{\left(\left\{B_{i}\right\},\left\{C_{j}\right\}\right)}^{k, l}$ to

$$
\left[\left.F\right|_{B_{1}}\right] \otimes \cdots \otimes\left[\left.F\right|_{B_{n-k-l}}\right] \otimes\left[\left.F\right|_{C_{1}}\right] \otimes \cdots \otimes\left[\left.F\right|_{C_{l}}\right]
$$

Here $\mathcal{V}(r, X)$ is defined in the same way as $\mathcal{V}(r, n)$ but with vertex set $X$, and $\left.F\right|_{X}$ means the tree in the forest $F$ whose vertex set is $X$. Now the stabilizer of $\left(\left\{B_{i}\right\},\left\{C_{j}\right\}\right)$ in $W(r, n)$ is a subgroup of the type mentioned in the statement, where $\lambda^{1}$ is formed from the sizes of the $B_{i}$ 's and $\lambda^{2}$ from the sizes of the $C_{j}$ 's. Thanks to (4) of Theorem 3.9, the action of this stabilizer on $\varepsilon_{n} \otimes A^{k, l}(T(r, n))_{\left(\left\{B_{i}\right\},\left\{C_{j}\right\}\right)}$ corresponds to the obvious action on the right-hand side of (3.4), together with a sign on the permutation of the open-root factors. The result follows.

Example 3.11. In the case $r=n=2$, Corollary 3.10 gives the following isomorphisms of representations of $W(2,2)$ :

$$
\begin{aligned}
& A^{0,0}(T(2,2)) \cong \varepsilon_{2} \otimes \operatorname{Ind}_{(W(2,1) \times W(2,1)) \rtimes S_{2}}^{W(2,2)}(\varepsilon \otimes \mathcal{V}(2,1) \otimes \mathcal{V}(2,1)) \cong 1, \\
& A^{1,0}(T(2,2)) \cong \varepsilon_{2} \otimes \operatorname{Ind}_{W(2,2)}^{W(2,2)}(\mathcal{V}(2,2)) \cong 1 \oplus \operatorname{prod}_{2}, \\
& A^{0,1}(T(2,2)) \cong \varepsilon_{2} \otimes \operatorname{Ind}_{W(2,1) \times W(2,1)}^{W(2,2)}(\mathcal{V}(2,1) \otimes \mathcal{V}(2,1)) \cong 1 \oplus \varepsilon_{2},
\end{aligned}
$$




$$
\begin{aligned}
& A^{1,1}(T(2,2)) \cong \varepsilon_{2} \otimes \operatorname{Ind}_{W(2,2)}^{W(2,2)}(\mathcal{V}(2,2)) \cong 1 \oplus \operatorname{prod}_{2}, \\
& A^{0,2}(T(2,2)) \cong \varepsilon_{2} \otimes \operatorname{Ind}_{(W(2,1) \times W(2,1)) \rtimes S_{2}}^{W(2,2)}(\mathcal{V}(2,1) \otimes \mathcal{V}(2,1)) \cong \varepsilon_{2} .
\end{aligned}
$$

Here we have used the obvious fact $\mathcal{V}(2,2) \cong \varepsilon_{2} \oplus \operatorname{det}_{2}$.

In general, we can substitute Corollary 2.10 into Corollary 3.10 to obtain:

Corollary 3.12. As a representation of $W(r, n), \varepsilon_{n} \otimes A^{k, l}(T(r, n))$ is isomorphic to the following direct sum:

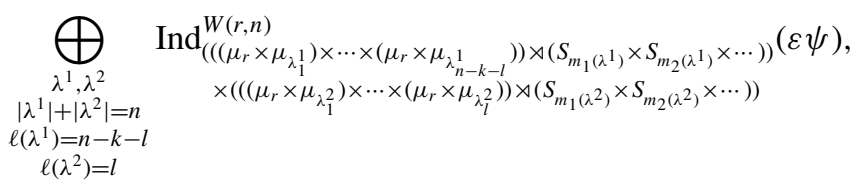

where $\psi$ is the character which takes the product of the $\mu_{\lambda_{a}^{j}}$ components, and $\varepsilon$ is the product of the sign characters of the $S_{m_{i}\left(\lambda^{1}\right)}$ components.

Summing over $k$ and $l$, we get an expression for $A^{\bullet}(T(r, n))$ as a direct sum of inductions of one-dimensional characters, which in the case $r=2$ is the same as $[3$, (1.1)].

Another consequence of Corollary 3.10 is a proof of the formula for the character of $H^{\bullet}(T(r, n), \mathbb{C})$ used in [6]. Since there are already at least three proofs of this character formula in the literature (Hanlon's original proof of the equivalent poset result in [4], Lehrer's proof in [10], and the 'equivariant inclusionexclusion' argument of [5, Theorem 9.4]), I will leave this to the reader's imagination.

\section{Cohomology of $T(1, n)$ with coefficients in a local system}

Now let $a_{1}, \ldots, a_{n} \in \mathbb{Q}$, and define the local system $\mathcal{L}_{\sum a_{i} \omega_{i}}$ on $T(1, n)$ as in the introduction. Let $r$ be a positive integer such that $r a_{i} \in \mathbb{Z}$ for all $i$. For any representation $V$ of $W(r, n)$, define

$$
V_{\left(a_{i}\right)}:=\left\{v \in V \mid \underline{\zeta} \cdot v=\zeta_{1}^{-r a_{1}} \cdots \zeta_{n}^{-r a_{n}} v, \forall \underline{\zeta} \in \mu_{r}^{n}\right\}
$$

It is clear that $V_{\left(a_{i}\right)}$ is stable under $Z_{\left(a_{i}\right)}$, the subgroup of $S_{n}$ which fixes $\left(a_{i}\right)$. If all $a_{i}$ equal $\frac{s}{r}$ for some $s \in \mathbb{Z}$, we write $V_{\left(a_{i}\right)}$ as $V_{\left(\frac{s}{r}\right)}$; in this case $Z_{\left(a_{i}\right)}$ is $S_{n}$ itself.

We are interested in $H^{\bullet}(T(r, n), \mathbb{C})_{\left(a_{i}\right)} \cong A^{\bullet}(T(r, n))_{\left(a_{i}\right)}$, which as mentioned in the Introduction is isomorphic to $H^{\bullet}\left(T(1, n), \mathcal{L}_{\sum a_{i} \omega_{i}}\right)$ via the map $\varphi: T(r, n) \rightarrow T(1, n)$ : $\left(z_{1}, \ldots, z_{n}\right) \mapsto\left(z_{1}^{r}, \ldots, z_{n}^{r}\right)$. To see this isomorphism explicitly, note that the pull祭 Springer 
back $\varphi^{*}: \Omega^{\bullet}(T(1, n)) \rightarrow \Omega^{\bullet}(T(r, n))$ sends $z_{i}$ to $z_{i}^{r}, d z_{i}$ to $r z_{i}^{r-1} d z_{i}$ and hence $\omega_{i}$ to $r \omega_{i}$ for all $i$. Recall that we have an isomorphism

$$
H^{p}\left(T(1, n), \mathcal{L}_{\sum a_{i} \omega_{i}}\right) \cong \frac{\operatorname{ker}\left(d+\ell_{\sum a_{i} \omega_{i}}: \Omega^{p}(T(1, n)) \rightarrow \Omega^{p+1}(T(1, n))\right)}{\operatorname{im}\left(d+\ell_{\sum a_{i} \omega_{i}}: \Omega^{p-1}(T(1, n)) \rightarrow \Omega^{p}(T(1, n))\right)},
$$

which is clearly $Z_{\left(a_{i}\right)}$-equivariant.

Proposition 4.1. The map $\alpha \mapsto z_{1}^{r a_{1}} \ldots z_{n}^{r a_{n}} \varphi^{*}(\alpha)$ defines an isomorphism of complexes $\left(\Omega^{\bullet}(T(1, n)), d+\ell_{\sum a_{i} \omega_{i}}\right) \stackrel{\sim}{\rightarrow}\left(\Omega^{\bullet}(T(r, n))_{\left(a_{i}\right)}, d\right)$, inducing $Z_{\left(a_{i}\right)}$-equivariant isoms $H^{p}\left(T(1, n), \mathcal{L}_{\sum a_{i} \omega_{i}}\right) \stackrel{\sim}{\rightarrow} H^{p}(T(r, n), \mathbb{C})_{\left(a_{i}\right)}$.

Proof: Since the action of $\mu_{r}^{n}$ on $\Omega^{\bullet}(T(r, n))$ respects $d$, the second statement follows automatically from the first. Now it is clear that $\varphi^{*}: \Omega^{0}(T(1, n)) \rightarrow \Omega^{0}(T(r, n))$ is an isomorphism onto the $\mu_{r}^{n}$-invariant subspace $\Omega^{0}(T(r, n))^{\mu_{r}^{n}}$. It follows that $\varphi^{*}: \Omega^{p}(T(1, n)) \stackrel{\sim}{\rightarrow} \Omega^{p}(T(r, n))^{\mu_{r}^{n}}$ for all $p$. Hence we have an isomorphism of complexes

$$
\varphi^{*}:\left(\Omega^{\bullet}(T(1, n)), d+\ell_{\sum a_{i} \omega_{i}}\right) \stackrel{\sim}{\rightarrow}\left(\Omega^{\bullet}(T(r, n))^{\mu_{r}^{n}}, d+\ell_{\sum r a_{i} \omega_{i}}\right) .
$$

Now for any $\alpha \in \Omega^{p}(T(r, n))$,

$$
\begin{aligned}
& z_{1}^{r a_{1}} \ldots z_{n}^{r a_{n}}\left(d+\ell_{\sum r a_{i} \omega_{i}}\right)(\alpha) \\
& \quad=z_{1}^{r a_{1}} \ldots z_{n}^{r a_{n}} d \alpha+\sum_{i} z_{1}^{r a_{1}} \ldots \widehat{z_{i}^{r a_{i}}} \ldots z_{n}^{r a_{n}} d\left(z_{i}^{r a_{i}}\right) \wedge \alpha \\
& \quad=d\left(z_{1}^{r a_{1}} \ldots z_{n}^{r a_{n}} \alpha\right) .
\end{aligned}
$$

Hence the map $\alpha \mapsto z_{1}^{r a_{1}} \ldots z_{n}^{r a_{n}} \alpha$ gives an isomorphism of complexes $\left.\left(\Omega^{\bullet}(T(r, n)), d+\ell_{\sum} r a_{i} \omega_{i}\right) \stackrel{\sim}{\rightarrow}\left(\Omega^{\bullet}(T \underset{\sim}{(r}, n)\right), d\right)$, which clearly restricts to an isomorphism $\left(\Omega^{\bullet}(T(r, n))^{\mu_{r}^{n}}, d+\ell_{\sum r a_{i} \omega_{i}}\right) \stackrel{\sim}{\rightarrow}\left(\Omega^{\bullet}(T(r, n))_{\left(a_{i}\right)}, d\right)$. Combining this with the isomorphism (4.1), we have the result.

In the previous section we saw that the structure of $A^{p}(T(r, n)) \cong H^{p}(T(r, n), \mathbb{C})$ was controlled by the forests in the set $\mathcal{F}(r, n)$. The main point of this section is that the structure of $A^{p}(T(r, n))_{\left(a_{i}\right)}$ is controlled by the forests in the following subset of $\mathcal{F}(1, n)$.

Definition 4.2. Let $\mathcal{F}\left(1, n ; a_{i}\right)$ be the set of $F \in \mathcal{F}(1, n)$ such that

$$
\sum_{i \in T} a_{i} \in \mathbb{Z}, \text { for all trees } T \text { in } F .
$$


Define $\mathcal{F}\left(1, n ; a_{i}\right)^{k, l}=\mathcal{F}\left(1, n ; a_{i}\right) \cap \mathcal{F}(1, n)^{k, l}$, and similarly $\mathcal{F}\left(1, n ; a_{i}\right)^{\circ}$ and $\mathcal{F}(1, n$; $\left.a_{i}\right)^{\circ, k, l}$.

Note that $\mathcal{F}\left(1, n ; a_{i}\right)$ is nonempty if and only if $\sum_{i} a_{i} \in \mathbb{Z}$. If all $a_{i}$ equal $\frac{s}{r}$, we write $\mathcal{F}\left(1, n ; a_{i}\right)$ as $\mathcal{F}\left(1, n ; \frac{s}{r}\right)$; it is then nonempty if and only if $r \mid s n$, i.e. $\frac{r}{\operatorname{gcd}(s, r)} \mid n$. Moreover, this being the case, $\mathcal{F}\left(1, n ; \frac{s}{r}\right)^{k, l}$ is nonempty if and only if $l \leq n-k \leq \frac{n \operatorname{gcd}(s, r)}{r}$, since the number of vertices in each tree must be a multiple of $\frac{r}{\operatorname{gcd}(s, r)}$.

Now for any $F \in \mathcal{F}(1, n)^{k, l}$, we define an element of $A^{k+l}(T(r, n))_{\left(a_{i}\right)}$ in the most obvious way:

$$
\beta(F):=\sum_{\underline{\zeta \in \mu_{r}^{n}}} \zeta_{1}^{r a_{1}} \ldots \zeta_{n}^{r a_{n}} \underline{\zeta} . \alpha(F)=\sum_{\underline{\zeta \in \mu_{r}^{n}}} \zeta_{1}^{r a_{1}} \ldots \zeta_{n}^{r a_{n}} \alpha(\underline{\zeta} . F)
$$

\section{Proposition 4.3.}

(1) $\beta(F) \neq 0 \Longleftrightarrow F \in \mathcal{F}\left(1, n ; a_{i}\right)$.

(2) $A^{\bullet}(T(r, n))_{\left(a_{i}\right)}$ has basis $\left\{\beta(F) \mid F \in \mathcal{F}\left(1, n ; a_{i}\right)^{\circ}\right\}$.

(3) For $w \in Z_{\left(a_{i}\right)}$ and $F \in \mathcal{F}\left(1, n ; a_{i}\right)$,

$$
w \cdot \beta(F)=\varepsilon_{n}(w) \varepsilon(w, F) \beta(w . F) .
$$

Proof: We know that the set $\left\{\alpha(\zeta . F) \mid \zeta \in \mu_{r}^{n}\right\}$ is linearly independent, although each element can correspond to many different $\zeta$. (If $F$ is rectified, this is part of the basis in Theorem 3.5, and the linear independence in general follows by applying a suitable element of $S_{n}$.) So it is clear that $\beta(F) \neq 0$ if and only if

$$
\sum_{\substack{\zeta \in \mu_{r}^{n} \\ \zeta . F=F}} \zeta_{1}^{r a_{1}} \ldots \zeta_{n}^{r a_{n}} \neq 0
$$

But the subgroup of $\mu_{r}^{n}$ fixing $F$ consists of the elements satisfying $\zeta_{i}=\zeta_{j}$ whenever $i$ and $j$ are vertices of the same tree in $F$. So the left-hand side of (4.4) factorizes into the product over the trees $T$ of $\sum_{\zeta \in \mu_{r}} \zeta^{\sum_{i \in T} r a_{i}}$, which is nonzero if and only if $r \mid \sum_{i \in T} r a_{i}$, i.e. $\sum_{i \in T} a_{i} \in \mathbb{Z}$. So (1) is proved. Now from Theorem 3.5 and the fact that any $F^{\prime} \in \mathcal{F}(r, n)^{\circ}$ is of the form $\zeta . F$ for some $F \in$ $\mathcal{F}(1, n)^{\circ}$, it is evident that $A^{\bullet}(T(r, n))_{\left(a_{i}\right)}$ is spanned by $\left\{\beta(F) \mid F \in \mathcal{F}(1, n)^{\circ}\right\}$. By (1), this means that $A^{\bullet}(T(r, n))_{\left(a_{i}\right)}$ is spanned by the nonzero elements $\{\beta(F) \mid F \in$ $\left.\mathcal{F}\left(1, n ; a_{i}\right)^{\circ}\right\}$. The latter elements are clearly linearly independent, as they involve disjoint collections of $\alpha\left(F^{\prime}\right)$ 's, so (2) is proved. Part (3) is a consequence of Proposition 3.7.

As a corollary, we have

$$
\operatorname{dim} A^{p}(T(r, n))_{\left(a_{i}\right)}=\sum_{k+l=p}\left|\mathcal{F}\left(1, n ; a_{i}\right)^{\circ, k, l}\right| .
$$


In particular, $A^{p}(T(r, n))_{\left(a_{i}\right)}=0$ unless $\sum_{i} a_{i} \in \mathbb{Z}$, and

$$
A^{p}(T(r, n))_{\left(\frac{s}{n}\right)} \neq 0 \Longleftrightarrow n-\operatorname{gcd}(s, n) \leq p \leq n .
$$

As described in the Introduction, we can write down explicit differential forms on $T(1, n)$ (not in the Orlik-Solomon algebra) which give the basis of $H^{p}\left(T(1, n), \mathcal{L}_{\sum a_{i} \omega_{i}}\right)$ corresponding under Proposition 4.1 to the basis $\{\beta(F) \mid F \in$ $\left.\bigcup_{k+l=p} \mathcal{F}\left(1, n ; a_{i}\right)^{\circ, k, l}\right\}$ of $A^{p}(T(r, n))_{\left(a_{i}\right)}$. For this we need some more notation associated with a forest $F \in \mathcal{F}\left(1, n ; a_{i}\right)$. Let $\simeq$ be the equivalence relation on $\{1, \ldots, n\}$ generated by $\rightarrow$, so $i \simeq j$ means that $i$ and $j$ are vertices of the same tree. Let $\preceq$ be the partial order generated by $\rightarrow$, so $i \preceq j$ means that there is a path from $i$ to $j$ traversing each edge in the chosen direction. Define

$$
\begin{aligned}
b_{j}(F) & =-\left\lfloor\sum_{k \leq j} a_{k}\right\rfloor+\sum_{i \rightarrow j}\left\lceil\sum_{k \preceq i} a_{k}\right\rceil \\
& =-\sum_{k \simeq j} a_{k}+\left\lceil\sum_{\substack{k \simeq j \\
k \preceq j}} a_{k}\right\rceil+\sum_{i \rightarrow j}\left\lceil\sum_{k \preceq i} a_{k}\right\rceil .
\end{aligned}
$$

(From the second expression it is clear that $b_{j}(F)$ is independent of the orientation of edges in $F$.) Then define

$$
\begin{aligned}
& \bar{\beta}(F):=\left(\prod_{j=1}^{n} z_{j}^{b_{j}(F)}\right) \bar{\beta}_{1}(F) \wedge \cdots \wedge \bar{\beta}_{n}(F) \in \Omega^{\bullet}(T(1, n)), \text { where } \\
& \bar{\beta}_{i}(F):= \begin{cases}\varepsilon_{i}(F) r, & \text { if } i \text { is an open root of } F, \\
\omega_{i}, & \text { if } i \text { is a closed root of } F, \\
\omega_{i, j}, & \text { if } i \rightarrow j \text { in } F \text { and } \sum_{k \leq i} a_{k} \in \mathbb{Z}, \\
\left(z_{i}-z_{j}\right)^{-1}\left(\omega_{i}-\omega_{j}\right), & \text { if } i \rightarrow j \text { in } F \text { and } \sum_{k \leq i} a_{k} \notin \mathbb{Z} .\end{cases}
\end{aligned}
$$

Note that $\left(z_{i}-z_{j}\right)^{-1}\left(\omega_{i}-\omega_{j}\right)=\frac{z_{j} d z_{i}-z_{i} d z_{j}}{z_{i} z_{j}\left(z_{i}-z_{j}\right)}$, and that $\sum_{k \leq i} a_{k} \in \mathbb{Z}$ if and only if the edge $i \rightarrow j$ is breakable, in the sense that the new trees formed by deleting it still satisfy (4.2).

Proposition 4.4. $\beta(F)=z_{1}^{r a_{1}} \ldots z_{n}^{r a_{n}} \varphi^{*}(\bar{\beta}(F)), \forall F \in \mathcal{F}\left(1, n ; a_{i}\right)$.

Proof: It is easy to see that $\beta(F)=\beta_{1}(F) \wedge \cdots \wedge \beta_{n}(F)$, where

$$
\beta_{i}(F)= \begin{cases}\varepsilon_{i}(F) r, & \text { if } i \text { is an open root of } F, \\ r \omega_{i}, & \text { if } i \text { is a closed root of } F, \\ \sum_{\eta \in \mu_{r}} \eta^{\sum_{k \leq i} r a_{k}} \omega_{i, j, \eta}, & \text { if } i \rightarrow j \text { in } F .\end{cases}
$$


The following can be proved by multiplying both sides by $z^{r}-w^{r}$ :

$$
\sum_{\eta \in \mu_{r}} \frac{\eta^{a}}{z-\eta w}=\frac{r z^{a-1-r\left\lfloor\frac{a-1}{r}\right\rfloor} w^{r-a+r\left\lfloor\frac{a-1}{r}\right\rfloor}}{z^{r}-w^{r}}, \text { for all } a \in \mathbb{Z} .
$$

Using this we obtain:

$$
\begin{aligned}
\sum_{\eta \in \mu_{r}} \eta^{a} \omega_{i, j, \eta} & = \begin{cases}\frac{r z_{i}^{a-r\left\lfloor\frac{a}{r}\right\rfloor} z_{j}^{r-a+r\left\lfloor\frac{a}{r}\right\rfloor}\left(\omega_{i}-\omega_{j}\right)}{z_{i}^{r}-z_{j}^{r}}, & \text { if } r \nmid a, \\
\frac{r\left(z_{i}^{r-1} d z_{i}-z_{j}^{r-1} d z_{j}\right)}{z_{i}^{r}-z_{j}^{r}}, & \text { if } r \mid a\end{cases} \\
& = \begin{cases}z_{i}^{a-r\left\lfloor\frac{a}{r}\right\rfloor} z_{j}^{-a+r\left\lceil\frac{a}{r}\right\rceil} \varphi^{*}\left(\left(z_{i}-z_{j}\right)^{-1}\left(\omega_{i}-\omega_{j}\right)\right), & \text { if } r \nmid a, \\
\varphi^{*}\left(\omega_{i, j}\right), & \text { if } r \mid a .\end{cases}
\end{aligned}
$$

So for any edge $i \rightarrow j$ in $F$,

$$
\beta_{i}(F)=z_{i}^{\sum_{k \leq i} r a_{k}-r\left\lfloor\sum_{k \leq i} a_{k}\right\rfloor} z_{j}^{-\sum_{k \leq i} r a_{k}+r\left\lceil\sum_{k \leq i} a_{k}\right\rceil} \varphi^{*}\left(\bar{\beta}_{i}(F)\right) .
$$

If $i$ is a root of $F$, we have

$$
\beta_{i}(F)=\varphi^{*}\left(\bar{\beta}_{i}(F)\right)=z_{i}^{\sum_{k \leq i} r a_{k}-r\left\lfloor\sum_{k \leq i} a_{k}\right\rfloor} \varphi^{*}\left(\bar{\beta}_{i}(F)\right),
$$

since in this case $\sum_{k \preceq i} a_{k} \in \mathbb{Z}$ by assumption. It only remains to note that for all $j$

$$
\sum_{k \preceq j} r a_{k}-r\left\lfloor\sum_{k \preceq j} a_{k}\right\rfloor-\sum_{i \rightarrow j} \sum_{k \preceq i} r a_{k}+\sum_{i \rightarrow j} r\left\lceil\sum_{k \preceq i} a_{k}\right\rceil=r a_{j}+r b_{j}(F)
$$

as required.

Combining Propositions 4.1, 4.3 and 4.4, we deduce the following.

Theorem 4.5. Let $H^{p}\left(1, n ; a_{i}\right)$ be the span of the differential forms $\bar{\beta}(F)$ for $F \in \bigcup_{k+l=p} \mathcal{F}\left(1, n ; a_{i}\right)^{k, l}$.

(1) $H^{p}\left(1, n ; a_{i}\right) \subset \operatorname{ker}\left(d+\ell_{\sum a_{i} \omega_{i}}: \Omega^{p}(T(1, n)) \rightarrow \Omega^{p+1}(T(1, n))\right)$.

(2) $H^{p}\left(1, n ; a_{i}\right) \stackrel{\sim}{\rightarrow} H^{p}\left(T(1, n), \mathcal{L}_{\sum} a_{i} \omega_{i}\right)$ via the map sending each form to its class $\bmod \operatorname{im}\left(d+\ell_{\sum a_{i} \omega_{i}}: \Omega^{p-1}(T(1, n)) \rightarrow \Omega^{p}(T(1, n))\right)$.

(3) $H^{p}\left(1, n ; a_{i}\right)$ has basis $\left\{\bar{\beta}(F) \mid F \in \bigcup_{k+l=p} \mathcal{F}\left(1, n ; a_{i}\right)^{\circ, k, l}\right\}$.

Springer 
(4) For $w \in Z_{\left(a_{i}\right)}$ and $F \in \mathcal{F}\left(1, n ; a_{i}\right)^{k, l}$,

$$
w \cdot \bar{\beta}(F)=\varepsilon_{n}(w) \varepsilon(w, F) \bar{\beta}(w \cdot F)
$$

Corollary 4.6. We have

$$
\operatorname{dim} H^{p}\left(T(1, n), \mathcal{L}_{\sum a_{i} \omega_{i}}\right)=\sum_{k+l=p}\left|\mathcal{F}\left(1, n ; a_{i}\right)^{\circ, k, l}\right|
$$

In particular, $H^{p}\left(T(1, n), \mathcal{L}_{\sum a_{i} \omega_{i}}\right)=0$ unless $\sum_{i} a_{i} \in \mathbb{Z}$, and

$$
H^{p}\left(T(1, n), \mathcal{L}_{\frac{s}{n}} \sum \omega_{i}\right) \neq 0 \Longleftrightarrow n-\operatorname{gcd}(s, n) \leq p \leq n .
$$

Example 4.7. If all $a_{i} \in \mathbb{Z}$ and $r=1, \bar{\beta}(F)=z_{1}^{-a_{1}} \cdots z_{n}^{-a_{n}} \alpha(F)$, and Theorem 4.5 is the obvious translation of the results for $H^{\bullet}(T(1, n), \mathbb{C})$ through the isomorphism $\mathcal{L}_{\sum a_{i} \omega_{i}} \cong \mathbb{C}$.

Example 4.8. If all $a_{i}=\frac{s}{n}$ and $\operatorname{gcd}(s, n)=1, \mathcal{F}\left(1, n ; \frac{s}{n}\right)$ is the union of $\mathcal{F}\left(1, n ; \frac{s}{n}\right)^{n-1,0}$ and $\mathcal{F}\left(1, n ; \frac{s}{n}\right)^{n-1,1}$. The former set consists of trees $T \in \mathcal{T}(1, n)$ with an open root; the second consists of the elements $T^{*}$, which are the trees $T \in \mathcal{T}(1, n)$ with a closed root. An easy calculation shows that for $T \in \mathcal{T}(1, n)^{\circ}$,

$$
\bar{\beta}(T)=\frac{(-1)^{n-1} n \prod_{j=1}^{n} z_{j}^{b_{j}(T)-1}}{p_{T}} \sum_{i}(-1)^{n-i} z_{i} d z_{1} \wedge \cdots \wedge \widehat{d z_{i}} \wedge \cdots \wedge d z_{n}
$$

whereas

$$
\bar{\beta}\left(T^{*}\right)=\frac{\prod_{j=1}^{n} z_{j}^{b_{j}(T)-1}}{p_{T}} d z_{1} \wedge \cdots \wedge d z_{n}
$$

If $s=-1$ we have $b_{j}(T)=1$ for all $j$, so the factors $\prod_{j=1}^{n} z_{j}^{b_{j}(T)-1}$ disappear. (Compare the basis of $A^{n-1}(M(n))$ given by (2.8).) Note that this example can be handled by more general methods, since $\frac{s}{n} \sum \omega_{i}-s \omega_{n}$ is not resonant (see Theorem 1.1). Indeed, Kawahara's result [7, Theorem 2.1] implies that $H^{p}\left(T(1, n), \mathcal{L}_{\frac{s}{n}} \sum \omega_{i}\right)=0$ for $p<n-1$, and [7, Theorem 2.3] provides another basis of the non-vanishing cohomologies.

Example 4.9. Take $n=4$, all $a_{i}=-\frac{1}{2}, \quad p=4$. The nine forests in $\bigcup_{k+l=4} \mathcal{F}\left(1,4 ;-\frac{1}{2}\right)^{\circ, k, l}$ are listed in the first column of the following table (asterisks indicate closed roots). The corresponding differential forms $\bar{\beta}(F)$, giving 
the basis of $H^{4}\left(1,4 ;-\frac{1}{2}\right)$, are in the second column.

$$
\begin{aligned}
& 1 \rightarrow 2 \rightarrow 3 \rightarrow 4^{*} \quad \frac{z_{2}}{\left(z_{1}-z_{2}\right)\left(z_{2}-z_{3}\right)\left(z_{3}-z_{4}\right)} d z_{1} \wedge d z_{2} \wedge d z_{3} \wedge d z_{4} \\
& 1 \rightarrow 2 \rightarrow 4^{*} \leftarrow 3 \quad \frac{z_{2}}{\left(z_{1}-z_{2}\right)\left(z_{2}-z_{4}\right)\left(z_{3}-z_{4}\right)} d z_{1} \wedge d z_{2} \wedge d z_{3} \wedge d z_{4} \\
& 1 \rightarrow 3 \rightarrow 4^{*} \leftarrow 2 \quad \frac{z_{3}}{\left(z_{1}-z_{3}\right)\left(z_{2}-z_{4}\right)\left(z_{3}-z_{4}\right)} d z_{1} \wedge d z_{2} \wedge d z_{3} \wedge d z_{4} \\
& 1 \rightarrow 4^{*} \leftarrow 3 \leftarrow 2 \quad \frac{z_{3}}{\left(z_{1}-z_{4}\right)\left(z_{2}-z_{3}\right)\left(z_{3}-z_{4}\right)} d z_{1} \wedge d z_{2} \wedge d z_{3} \wedge d z_{4} \\
& \begin{array}{l}
\uparrow \rightarrow 4^{*} \\
2
\end{array} \rightarrow \frac{z_{3}}{\left(z_{1}-z_{3}\right)\left(z_{2}-z_{3}\right)\left(z_{3}-z_{4}\right)} d z_{1} \wedge d z_{2} \wedge d z_{3} \wedge d z_{4} \\
& 1 \rightarrow 4^{*} \leftarrow 3 \\
& \uparrow \quad \frac{z_{4}}{\left(z_{1}-z_{4}\right)\left(z_{2}-z_{4}\right)\left(z_{3}-z_{4}\right)} d z_{1} \wedge d z_{2} \wedge d z_{3} \wedge d z_{4} \\
& 1 \rightarrow 2^{*} \quad 3 \rightarrow 4^{*} \quad \frac{1}{\left(z_{1}-z_{2}\right)\left(z_{3}-z_{4}\right)} d z_{1} \wedge d z_{2} \wedge d z_{3} \wedge d z_{4} \\
& 1 \rightarrow 3^{*} \quad 2 \rightarrow 4^{*} \quad \frac{1}{\left(z_{1}-z_{3}\right)\left(z_{2}-z_{4}\right)} d z_{1} \wedge d z_{2} \wedge d z_{3} \wedge d z_{4} \\
& 1 \rightarrow 4^{*} \quad 2 \rightarrow 3^{*} \quad \frac{1}{\left(z_{1}-z_{4}\right)\left(z_{2}-z_{3}\right)} d z_{1} \wedge d z_{2} \wedge d z_{3} \wedge d z_{4}
\end{aligned}
$$

The wedge product of differential forms endows $H^{\bullet}(T(1, n), \mathbb{C})$ with a ring structure, and $H^{\bullet}\left(T(1, n), \mathcal{L}_{\omega}\right)$ with the structure of $H^{\bullet}(T(1, n), \mathbb{C})$-module for any $\omega \in A^{1}(T(1, n))$. A consequence of Theorem 4.5 is:

Corollary 4.10. The $H^{\bullet}(T(1, n), \mathbb{C})$-module $H^{\bullet}\left(T(1, n), \mathcal{L}_{\sum a_{i} \omega_{i}}\right)$ is generated by the images of those $\bar{\beta}(F)$ 's where $F \in \mathcal{F}\left(1, n ; a_{i}\right)^{\circ}$ has no closed roots or breakable edges. In particular, $H^{\bullet}\left(T(1, n), \mathcal{L}_{\frac{s}{n}} \sum \omega_{i}\right)$ is generated by $H^{n-\operatorname{gcd}(s, n)}\left(T(1, n), \mathcal{L}_{\frac{s}{n}} \sum \omega_{i}\right)$.

Proof: For general $F \in \mathcal{F}\left(1, n ; a_{i}\right)^{\circ}$, let $\widetilde{F} \in \mathcal{F}\left(1, n ; a_{i}\right)^{\circ}$ be the forest obtained by deleting all breakable edges and opening all closed roots. It is easy to see that $b_{j}(F)=$ $b_{j}(\widetilde{F})$ for all $j$, so $\bar{\beta}(F)$ is, up to scalar, the wedge product of $\bar{\beta}(\widetilde{F})$ with a collection of 1-forms $\omega_{i}$ and $\omega_{i, j}$. Since these 1-forms are closed, the first statement follows. In the special case when all $a_{i}=\frac{s}{n}$, it is clear that $\widetilde{F} \in \mathcal{F}\left(1, n ; a_{i}\right)^{\circ, n-\operatorname{gcd}(s, n), 0}$, whence the second statement. 
It would be interesting to find more information about this module, such as a projective resolution.

Our remaining goal is to describe $H^{\bullet}\left(T(1, n), \mathcal{L}_{\sum a_{i} \omega_{i}}\right)$ as a graded representation of $Z_{\left(a_{i}\right)}$. It is more convenient to describe the isomorphic representation $A^{\bullet}(T(r, n))_{\left(a_{i}\right)}$; all results can be translated easily using Propositions 4.1 and 4.4.

We start with the analogue of Proposition 3.8.

Proposition 4.11. The following hold in $A^{\bullet}(T(r, n))_{\left(a_{i}\right)}$.

(1) $\beta\left(F_{1}\right)+\beta\left(F_{2}\right)=\beta\left(F_{3}\right)$, whenever $F_{1}, F_{2}, F_{3} \in \mathcal{F}\left(1, n ; a_{i}\right)$ are as in (1) of Proposition 3.8.

(2) $\beta(F)+\beta\left(F^{\prime}\right)=0$, whenever $F, F^{\prime} \in \mathcal{F}\left(1, n ; a_{i}\right)$ are as in (2) of Proposition 3.8.

(3) $\beta(F)+\beta\left(F^{\prime}\right)=\beta\left(F^{\prime \prime}\right)$ whenever $F, F^{\prime}, F^{\prime \prime} \in \mathcal{F}\left(1, n ; a_{i}\right)$ are as in (3) of Proposition 3.8.

(4) $\beta(F)+\beta\left(F^{\prime}\right)=0$ whenever $F, F^{\prime} \in \mathcal{F}\left(1, n ; a_{i}\right)$ are as in (3) of Proposition 3.8 and the corresponding $F^{\prime \prime}$ is not in $\mathcal{F}\left(1, n ; a_{i}\right)$.

Proof: In each case (1)-(3), the translates of the forests by a fixed $\zeta \in \mu_{r}^{n}$ are still a triple/pair of the right form, so these all follow instantly from Proposition 3.8. The same applies in case (4), except that $\beta\left(F^{\prime \prime}\right)=0$ by (1) of Proposition 4.3.

The analogue of Theorem 3.9 follows, with an entirely analogous proof. For any $F \in \mathcal{F}\left(1, n ; a_{i}\right)^{k, l}$, let $\langle F\rangle$ denote the image of $\beta(F)$ in

$$
A^{k, l}(T(r, n))_{\left(a_{i}\right)}=\left(A^{k+l}(T(r, n))_{l}\right)_{\left(a_{i}\right)} /\left(A^{k+l}(T(r, n))_{l+1}\right)_{\left(a_{i}\right)} .
$$

Theorem 4.12. With notation as above:

(1) $A^{p}(T(r, n))_{\left(a_{i}\right)}$ can be defined abstractly as the vector space spanned by $\left\{\beta(F) \mid F \in \bigcup_{k+l=p} \mathcal{F}\left(1, n ; a_{i}\right)^{k, l}\right\}$ subject to the relations in Proposition 4.11 .

(2) $A^{k, l}(T(r, n))_{\left(a_{i}\right)}$ can be defined abstractly as the vector space spanned by $\{\langle F\rangle \mid F \in$ $\left.\mathcal{F}\left(1, n ; a_{i}\right)^{k, l}\right\}$ subject to the following relations:

- $\left\langle F_{1}\right\rangle+\left\langle F_{2}\right\rangle=\left\langle F_{3}\right\rangle$ whenever $F_{1}, F_{2}, F_{3} \in \mathcal{F}\left(1, n ; a_{i}\right)^{k, l}$ are as in (1) of Proposition 3.8;

- $\langle F\rangle+\left\langle F^{\prime}\right\rangle=0$ whenever $F, F^{\prime} \in \mathcal{F}\left(1, n ; a_{i}\right)^{k, l}$ are as in (2) or (3) of Proposition 3.8 .

(3) $A^{k, l}(T(r, n))_{\left(a_{i}\right)}$ has basis $\left\{\langle F\rangle \mid F \in \mathcal{F}\left(1, n ; a_{i}\right)^{\circ, k, l}\right\}$.

(4) For $w \in Z_{\left(a_{i}\right)}$ and $F \in \mathcal{F}\left(1, n ; a_{i}\right)^{k, l}$,

$$
w \cdot\langle F\rangle=\varepsilon_{n}(w) \varepsilon(w, F)\langle w . F\rangle .
$$

(5) As a representation of $Z_{\left(a_{i}\right)}$,

$$
A^{p}(T(r, n))_{\left(a_{i}\right)} \cong \bigoplus_{k+l=p} A^{k, l}(T(r, n))_{\left(a_{i}\right)} .
$$


For the remaining results we restrict to the case where all $a_{i}=\frac{s}{r}, \operatorname{gcd}(s, r)=1$, and $r \mid n$. We have an analogue of Corollary 3.10, proved in the same way (incorporating the extra feature that all trees must have a number of vertices divisible by $r$ ):

Corollary 4.13. As a representation of $S_{n}, \varepsilon_{n} \otimes A^{k, l}(T(r, n))_{\left(\frac{s}{r}\right)}$ is isomorphic to the following direct sum:

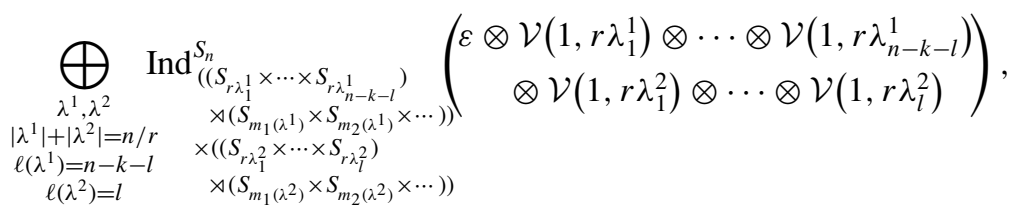

where $S_{r \lambda_{a}^{j}}$ acts on the $\mathcal{V}\left(1, r \lambda_{a}^{j}\right)$ factor, $S_{m_{i}\left(\lambda^{j}\right)}$ acts by permuting the $\mathcal{V}\left(1, r \lambda_{a}^{j}\right)$ factors where $\lambda_{a}^{j}=i$, and $\varepsilon$ denotes the product of the sign characters of the $S_{m_{i}\left(\lambda^{1}\right)}$ components.

Example 4.14. If $r=n$, Corollary 4.13 says that $\varepsilon_{n} \otimes A^{n-1,0}(T(n, n))_{\left(\frac{s}{n}\right)}$ and $\varepsilon_{n} \otimes$ $A^{n-1,1}(T(n, n))_{\left(\frac{s}{n}\right)}$ are isomorphic to $\mathcal{V}(1, n)$.

Example 4.15. In the case $n=4, r=2, s=1$, Corollary 4.13 gives the following isomorphisms of representations of $S_{4}$ :

$$
\begin{aligned}
& A^{2,0}(T(2,4))_{\left(\frac{1}{2}\right)} \cong \varepsilon_{4} \otimes \operatorname{Ind}_{\left(S_{2} \times S_{2}\right) \rtimes S_{2}}^{S_{4}}(\varepsilon \otimes \mathcal{V}(1,2) \otimes \mathcal{V}(1,2)) \cong V^{(31)}, \\
& A^{3,0}(T(2,4))_{\left(\frac{1}{2}\right)} \cong \varepsilon_{4} \otimes \operatorname{Ind}_{S_{4}}^{S_{4}}(\mathcal{V}(1,4)) \cong V^{(31)} \oplus V^{\left(21^{2}\right)}, \\
& A^{2,1}(T(2,4))_{\left(\frac{1}{2}\right)} \cong \varepsilon_{4} \otimes \operatorname{Ind}_{S_{2} \times S_{2}}^{S_{4}}(\mathcal{V}(1,2) \otimes \mathcal{V}(1,2)) \cong V^{(4)} \oplus V^{(31)} \oplus V^{\left(2^{2}\right)}, \\
& A^{3,1}(T(2,4))_{\left(\frac{1}{2}\right)} \cong \varepsilon_{4} \otimes \operatorname{Ind}_{S_{4}}^{S_{4}}(\mathcal{V}(1,4)) \cong V^{(31)} \oplus V^{\left(21^{2}\right)}, \\
& A^{2,2}(T(2,4))_{\left(\frac{1}{2}\right)} \cong \varepsilon_{4} \otimes \operatorname{Ind}_{\left(S_{2} \times S_{2}\right) \rtimes S_{2}}^{S_{4}}(\mathcal{V}(1,2) \otimes \mathcal{V}(1,2)) \cong V^{(4)} \oplus V^{\left(2^{2}\right)} .
\end{aligned}
$$

Here the irreducible representations of $S_{4}$ are denoted $V^{\lambda}$, where $\lambda$ is a partition of 4; the convention is the one where $V^{(4)} \cong 1, V^{\left(1^{4}\right)} \cong \varepsilon_{4}$.

We can substitute Theorem 2.9 into Corollary 4.13, to obtain:

Corollary 4.16. As a representation of $S_{n}, \varepsilon_{n} \otimes A^{k, l}(T(r, n))_{\left(\frac{s}{r}\right)}$ is isomorphic to the following direct sum:

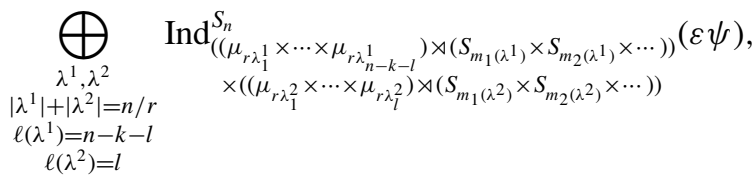


where $\psi$ is the character which takes the product of the $\mu_{r \lambda_{a}^{j}}$ components, and $\varepsilon$ is the product of the sign characters of the $S_{m_{i}\left(\lambda^{1}\right)}$ components.

Summing over $k$ and $l$, we get the desired expression for $A^{\bullet}(T(r, n))_{\left(\frac{s}{r}\right)} \cong$ $H^{\bullet}(T(r, n), \mathbb{C})_{\left(\frac{s}{r}\right)} \cong H^{\bullet}\left(T(1, n), \mathcal{L}_{\frac{s}{r}} \sum \omega_{i}\right)$ as a direct sum of inductions of onedimensional characters.

Furthermore, there is an obvious relationship between Corollaries 3.10 and 4.13, which gives the following explanation of (1.3).

\section{Corollary 4.17. As a representation of $S_{n}$,}

$$
\varepsilon_{n} \otimes A^{k, l}(T(r, n))_{\left(\frac{s}{r}\right)} \cong \operatorname{Ind}_{W(r, n / r)}^{S_{n}}\left(\operatorname{det}_{n / r} \otimes A^{k-n+n / r, l}(T(r, n / r))\right) .
$$

Proof: For the left-hand side, use the expression of Corollary 4.13, with the righthand expression of Theorem 2.11 substituted for each of the $\mathcal{V}\left(1, r \lambda_{a}^{j}\right)$ factors. For the right-hand side, use the expression given by Corollary 3.10. Comparing, we get the result.

Acknowledgements I am very grateful to H. Barcelo, G. Lehrer, and the referees for their helpful comments on this work.

\section{References}

1. H. Barcelo, "On the action of the symmetric group on the free Lie algebra and the partition lattice," $J$. Combin. Theory Ser. A, 55 (1990), 93-129.

2. D.C. Cohen, "Triples of arrangements and local systems," Proc. Amer. Math. Soc. 130(10) (2002), 3025-3031.

3. J.M. Douglass, "On the cohomology of an arrangement of type $B_{l}$," J. Algebra 147 (1992), 265-282.

4. P. Hanlon, "The characters of the wreath product group acting on the homology groups of the Dowling lattices," J. Algebra 91 (1984), 430-463.

5. A. Henderson, "Representations of wreath products on cohomology of De Concini-Procesi compactifications," Int. Math. Res. Not. 20 (2004), 983-1021.

6. A. Henderson, "The symmetric group representation on cohomology of the regular elements of a maximal torus of the special linear group," math.RT/0312006.

7. Y. Kawahara, "Vanishing and bases for cohomology of partially trivial local systems on hyperplane arrangements," Proc. Amer. Math. Soc. 133(7) (2005), 1907-1915.

8. G.I. Lehrer, "On the Poincaré series associated with Coxeter group actions on complements of hyperplanes," J. London Math. Soc. 36(2) (1987), 275-294.

9. G.I. Lehrer, "On hyperoctahedral hyperplane complements," in The Arcata Conference on Representations of Finite Groups (Arcata, Calif., 1986), Vol. 47 of Proc. Sympos. Pure Math., Amer. Math. Soc., Providence, RI, 1987, pp. 219-234.

10. G.I. Lehrer, "Poincaré polynomials for unitary reflection groups," Invent. Math. 120 (1995), 411-425.

11. G.I. Lehrer and L. Solomon, "On the action of the symmetric group on the cohomology of the complement of its reflecting hyperplanes," J. Algebra. 104 (1986), 410-424.

12. A. Libgober and S. Yuzvinsky, "Cohomology of local systems," in Arrangements-Tokyo 1998, Vol. 27 of Adv. Stud. Pure Math., Kinokuniya, Tokyo, 2000, pp. 169-184. 
13. P. Orlik and H. Terao, "Arrangements of Hyperplanes. Springer-Verlag, 1992.

14. V. Schechtman, H. Terao, and A. Varchenko, "Local systems over complements of hyperplanes and the Kac-Kazhdan conditions for singular vectors," J. Pure Appl. Algebra 100(1-3) (1995), 93102.

15. S. Yuzvinsky, "Orlik-Solomon algebras in algebra and topology," Uspekhi Mat. Nauk 56(2) (2001), (338), pp. 87-166, translation in Russian Math. Surveys 56(2) (2001), 293-364. 